\title{
X-ray diffraction microscopy based on refractive optics
}

Poulsen, Henning Friis; Jakobsen, A. C.; Simons, Hugh; Ahl, Sonja Rosenlund; Cook, P. K.; Detlefs, C.

Published in:

Journal of Applied Crystallography

Link to article, DOI:

$10.1107 /$ S1600576717011037

Publication date:

2017

Document Version

Publisher's PDF, also known as Version of record

Link back to DTU Orbit

Citation (APA):

Poulsen, H. F., Jakobsen, A. C., Simons, H., Ahl, S. R., Cook, P. K., \& Detlefs, C. (2017). X-ray diffraction microscopy based on refractive optics. Journal of Applied Crystallography, 50(5), 1441-1456.

https://doi.org/10.1107/S1600576717011037

\section{General rights}

Copyright and moral rights for the publications made accessible in the public portal are retained by the authors and/or other copyright owners and it is a condition of accessing publications that users recognise and abide by the legal requirements associated with these rights.

- Users may download and print one copy of any publication from the public portal for the purpose of private study or research.

- You may not further distribute the material or use it for any profit-making activity or commercial gain

- You may freely distribute the URL identifying the publication in the public portal 


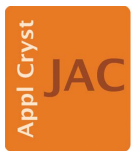

JOURNAL OF APPLIED CRYSTALLOGRAPHY

ISSN 1600-5767

Received 8 May 2017

Accepted 26 July 2017

Edited by G. Kostorz, ETH Zurich, Switzerland

Keywords: X-ray diffraction microscopy; diffraction contrast tomography; structural characterization; synchrotron radiation; diffraction imaging.

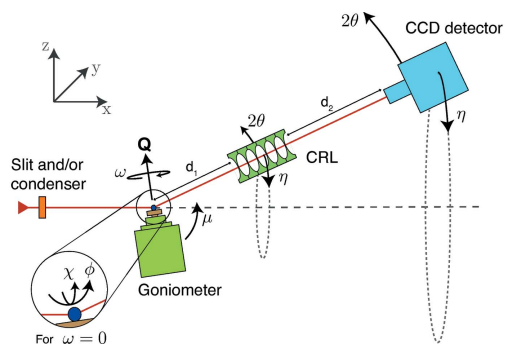

C 2017 International Union of Crystallography

\section{X-ray diffraction microscopy based on refractive optics}

\author{
H. F. Poulsen, ${ }^{a *}$ A. C. Jakobsen, ${ }^{a}$ H. Simons, ${ }^{a}$ S. R. Ahl, ${ }^{a}$ P. K. Cook ${ }^{b}$ and C. Detlefs ${ }^{b}$

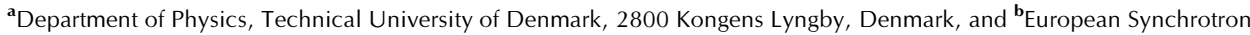 \\ Radiation Facility, 71 avenue des Martyrs, CS40220, 38043 Grenoble Cedex 9, France. *Correspondence e-mail: \\ hfpo@fysik.dtu.dk
}

A formalism is presented for dark-field X-ray microscopy using refractive optics. The new technique can produce three-dimensional maps of lattice orientation and axial strain within millimetre-sized sampling volumes and is particularly suited to in situ studies of materials at hard X-ray energies. An objective lens in the diffracted beam magnifies the image and acts as a very efficient filter in reciprocal space, enabling the imaging of individual domains of interest with a resolution of $100 \mathrm{~nm}$. Analytical expressions for optical parameters such as numerical aperture, vignetting, and the resolution in both direct and reciprocal spaces are provided. It is shown that the resolution function in reciprocal space can be highly anisotropic and varies as a function of position in the field of view. Inserting a square aperture in front of the objective lens facilitates disjunct and space-filling sampling, which is key for three-dimensional reconstruction and analysis procedures based on the conservation of integrated intensity. A procedure for strain scanning is presented. Finally the formalism is validated experimentally at an X-ray energy of $17 \mathrm{keV}$.

\section{Introduction}

Hard X-ray microscopy is a new full-field imaging technique for mapping bulk specimens in three dimensions. In brightfield mode, an X-ray objective is placed in the transmitted beam. The attenuation and refraction of the incoming beam is monitored, leading to magnified absorption and phase contrast reconstructions. In the dark-field mode, which is the topic of this article, the objective is placed in the Bragg diffracted beam instead. This enables nondestructive mapping of the structure, orientation and strain of deeply embedded crystalline elements (Simons et al., 2015). The magnification and field of view can be modified by changing the focal length of the objective lens. The objective only allows diffraction signals to pass to the detector if the scattering vector is in the vicinity of a nominal point in reciprocal space. Hence, it acts as a very effective filter for stray diffraction signals, thereby facilitating studies of individual domains by suppressing unwanted overlap of diffraction signals.

A first implementation at beamline ID06 at the European Synchrotron (ESRF) is based on the use of a monochromatic beam in the $15-35 \mathrm{keV}$ range. Here the technique is combined with coarse-scale three-dimensional grain mapping techniques such as three-dimensional X-ray diffraction (3DXRD) (Poulsen et al., 2001; Poulsen, 2012; Hefferan et al., 2012; Schmidt, 2014) and diffraction contrast tomography (DCT) (King et al., 2008; Ludwig et al., 2009), as well as classical tomography. The orientation matrices from 3DXRD or DCT can be imported directly into the microscopy software. This 
enables swapping between fast overviews of the entire specimen on the grain scale and detailed studies of domains within selected grains without dismounting the sample. First applications include work on the processing of plastically deformed metals (Ahl et al., 2015), the distribution of strain and orientation gradients in ferroelectrics (Simons, Jakobsen et al., 2016) and the three-dimensional mapping of dislocations (Jakobsen et al., 2017).

The objective is in the current implementation of the microscope a compound refractive lens (CRL) (Snigirev et al., 1996). Refractive X-ray optics have relatively small numerical apertures (NA) of NA $\leq 10^{-3}$. The diffraction limit implies that the spatial resolution $\Delta r \geq \lambda / \mathrm{NA}$. In practice manufacturing errors further limit the spatial resolution to $\sim 50$ $100 \mathrm{~nm}$. As we shall demonstrate in this paper, orientation and strain can be mapped with a sensitivity of $0.1 \mathrm{mrad}$ and $10^{-4}$, respectively - superior to transmission electron microscopy (Williams \& Carter, 2009). Furthermore, having the ability to change the focal length of a CRL enables zooming in and out of both direct and reciprocal space, thereby realizing a compromise between image acquisition time and spatial and angular sensitivity. The coupling between direct and reciprocal space through the numerical aperture implies that a full description of dark-field X-ray microscopy requires a formulation in six-dimensional position-reciprocal space (see also Poulsen, 2003).

In this paper we provide analytical expressions for the key parameters in dark-field X-ray microscopy, such as the numerical aperture, the field of view, the depth of field, and the resolution in direct and reciprocal space. We discuss the implications for mapping of strain and orientation, and present sampling procedures for three-dimensional mapping of extended regions in both direct and reciprocal space. The expressions presented are based on a generalization of a recent thick-lens ray-transfer-matrix description of CRLs established for bright-field microscopy (Simons et al., 2017).

\section{Geometry of dark-field microscopy}

The geometry of dark-field X-ray microscopy is illustrated in Fig. 1. The sample goniometer has a base tilt, $\mu$, a rotation, $\omega$, and two orthogonal sample tilts, $\chi$ and $\phi$. The incident beam is typically shaped by a condenser and characterized by angular divergences, $\Delta \zeta_{\mathrm{v}}$ and $\Delta \zeta_{\mathrm{h}}$, in the vertical and horizontal directions, respectively, and by an energy bandwidth $\Delta E / E$. The motors $\chi, \phi$ and $\mu$ are used to orient an embedded crystalline element of choice (e.g. a grain or domain) such that it is in the Laue condition with its scattering vector, $\mathbf{Q}$, parallel to the rotation axis $\omega$, implying that $\mathbf{Q}$ remains in the diffraction condition at all values of $\omega$ (for details see Appendix A).

The optical axis of the diffracted beam is defined by the centre of rotation of the sample goniometer, the centre of the objective and the point of normal incidence of the beam on the detector. The direction of this axis is described by the scattering angle, $2 \theta$, and the azimuthal angle, $\eta$ (Fig. 1). The objective magnifies the diffracted beam by a factor $\mathcal{M}_{\mathrm{CRL}}$ and generates an inverted two-dimensional image on the detector. The distance from the sample plane to the front of the objective is $d_{1}$ and the distance between sample plane and image plane is $L=d_{1}+N T+d_{2}$, where $N T$ is the length of the CRL (the product of the number of lenses $N$ and the distance $T$ between the centres of adjacent lenslets).

There are two alternative strategies for obtaining threedimensional maps of real-space distributions. The first is by using a one-dimensionally focusing condenser to illuminate a slice of the material, which is then imaged at the oblique angle of $2 \theta_{\mathrm{B}}$, i.e. a magnified version of classical section topography (Medrano et al., 1997; Ohler et al., 2000). In this case, a threedimensional volume is obtained in a layer-wise manner by translating the sample through the planar beam in small increments. A second, faster, but more involved method involves illuminating the entire grain and taking projections from different viewing angles while rotating the sample about Q (i.e. rotation in $\omega$ ) in the topo-tomography approach (Ludwig et al., 2001). The three-dimensional maps are then reconstructed using adapted tomographic algorithms. The three-dimensional reconstruction algorithm itself, however, is outside the scope of this paper.

For a given layer or a given projection, the local variation in orientation of the scattering vector (local pole figure) can be mapped in two ways: first, by scanning the sample through $(\phi, \chi)$ or linear combinations of these angles, and second, by scanning a combination of the base tilt $\mu$ and a linear combination of $(\phi, \chi)$ that is perpendicular to the beam for all values of $\omega$. The optimal choice of method and linear combination depends on motor accuracy and speed. Maps of the axial strain can be acquired by a combined scan of the optical axis (i.e. the objective and detector collectively) through $2 \theta$ and of the sample by an angle $\theta$, with the rotation axis of $2 \theta$ and $\theta$ being collinear.

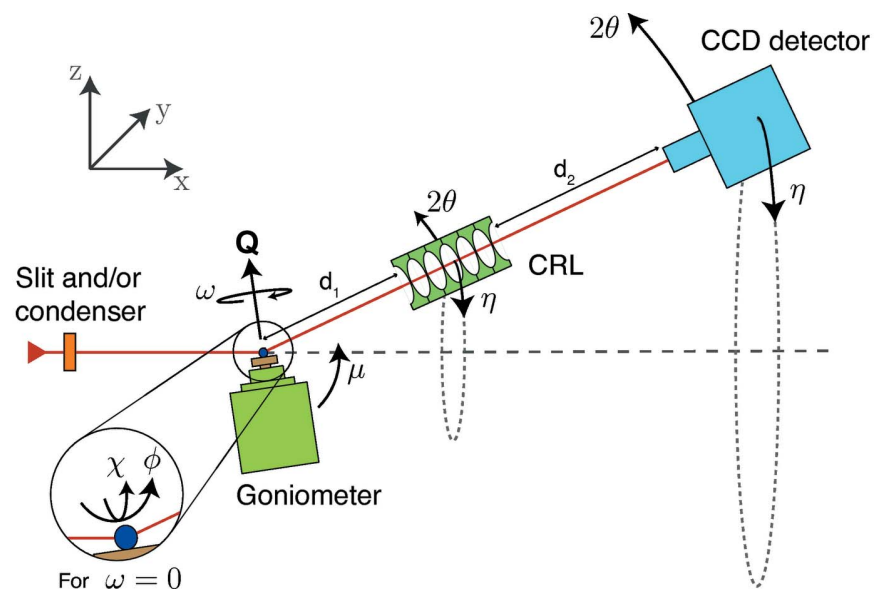

Figure 1

Principle of dark-field X-ray microscopy. The red line between the pivotal point of the goniometer and the detector is the optical axis of the diffracted beam. A laboratory coordinate system is defined with $x$ parallel to the incoming beam, $y$ horizontal and $z$ vertical. See also main text and Fig. 7. 


\subsection{Coordinate systems}

The instrumental setting can be related to the reciprocal space by coordinate transforms and crystallographic relationships, similar to the work by Busing \& Levy (1967) and You (1999). A comprehensive treatment is presented in Appendix $A$, with reference to the hard X-ray microscope (HXRM) at the ESRF. For simplicity, in the main text, we will assume that the detector is positioned in the centre of the vertical scattering plane $(\eta=0)$ and that the base tilt is fixed at $\mu=\theta_{\mathrm{B}}$, the nominal Bragg angle. In this configuration the position in reciprocal space is given by the four motor positions: $\chi, \phi, 2 \theta$ and $\omega$. Note that, when $\omega=0, \phi$ represents the 'rocking' of the sample around the laboratory $y$ axis, and $\chi$ the 'rolling' around an axis within the scattering plane and perpendicular to $\mathbf{Q}$ ( $c f$. Fig. 1).

Dark-field microscopy images the diffracted intensity in close proximity to one scattering vector $\mathbf{Q}_{0}$, parameterized by angles $\left(\phi_{0}, \chi_{0}, 2 \theta_{\mathrm{B}}\right)$. In order to describe small variations from this nominal scattering vector, it is useful to introduce a local coordinate system in reciprocal space, denoted the 'reference system' (see Appendix $A$ ): $\mathbf{Q}=\Delta Q_{\text {rock }} \hat{q}_{\text {rock }}+\Delta Q_{\text {roll }} \hat{q}_{\text {roll }}+$ $\Delta Q_{\|} \hat{q}_{\|}$, as shown in Fig. 2. Here, $\hat{q}_{\|}$is parallel to $\mathbf{Q}_{0}$, while $\hat{q}_{\text {roll }}$ is parallel to the laboratory $y$ axis for $\omega=0$. The appendix provides the relevant coordinate transforms. It is shown that for small angles $\left|\phi-\phi_{0}\right|$ and $\left|\chi-\chi_{0}\right|$ we can associate motor positions $(\chi, \phi, 2 \theta, \omega)$ with a position in the reciprocal space as follows:

$$
\begin{aligned}
\frac{\Delta Q_{\text {rock }}}{\left|\mathbf{Q}_{0}\right|}= & -\frac{2 \theta-2 \theta_{\mathrm{B}}}{2}-\sin (\omega)\left(\chi-\chi_{0}\right) \\
& +\cos (\chi) \cos (\omega)\left(\phi-\phi_{0}\right),
\end{aligned}
$$

$$
\frac{\Delta Q_{\text {roll }}}{\left|\mathbf{Q}_{0}\right|}=\cos (\omega)\left(\chi-\chi_{0}\right)+\cos (\chi) \sin (\omega)\left(\phi-\phi_{0}\right),
$$

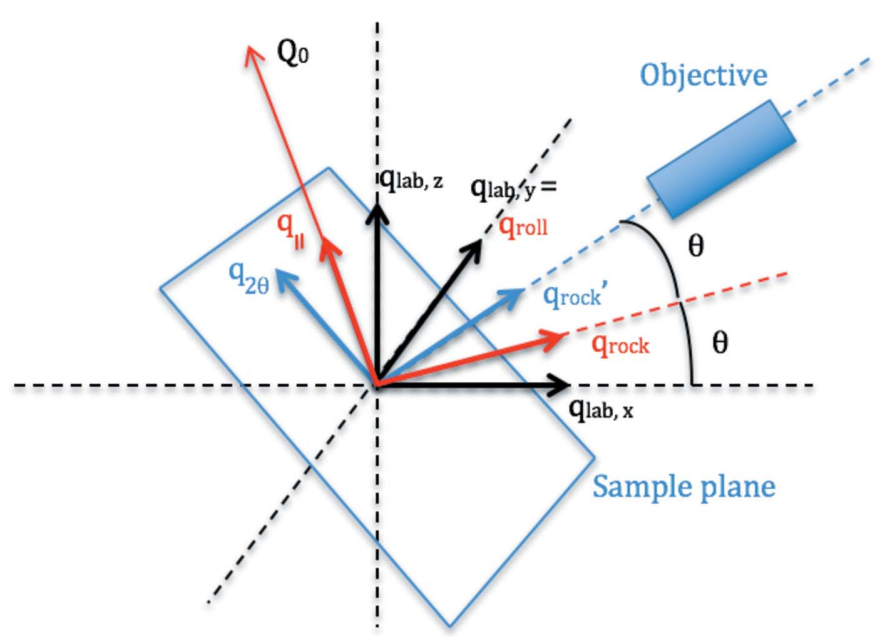

Figure 2

Definition of the reference $\left(\hat{q}_{\text {rock }}, \hat{q}_{\text {roll }}, \hat{q}_{\|}\right)$and imaging coordinate systems $\left(\hat{q}_{\text {rock' }}, \hat{q}_{\text {roll }}, \hat{q}_{2 \theta}\right)$ and their relation to the laboratory system $\left(\hat{q}_{\mathrm{lab}, x}, \hat{q}_{\mathrm{lab}, y}, \hat{q}_{\mathrm{lab}, z}\right)$ for $\omega=0$. All vectors except $\mathbf{Q}_{0}$ are unit vectors and all vectors except $\hat{q}_{\text {lab }, y}=\hat{q}_{\text {roll }}$ are in the same plane. The sample plane normal is $\hat{q}_{\text {rock' }}$.

$$
\frac{\Delta Q_{\|}}{\left|\mathbf{Q}_{0}\right|}=\frac{2 \theta-2 \theta_{\mathrm{B}}}{2 \tan \left(\theta_{\mathrm{B}}\right)} .
$$

The first term in equation (1) reflects the well known fact that a longitudinal scan requires the rocking angle to be changed by half the change in the scattering angle. Numerical tests show that these equations are accurate up to angles of at least $5^{\circ}$.

It is also useful to introduce an 'imaging coordinate system' with the direction of the optical axis as the $x$ axis. In direct space the plane perpendicular to this axis is the sample plane, while in reciprocal space it is the tangential plane of the Ewald sphere at point $\mathbf{Q}_{0}$. At $\omega=0$ the laboratory system, the reference system and the imaging system are related by rotations of $\theta_{\mathrm{B}}$ around the $y$ axis, as illustrated in Fig. 2.

\subsection{Geometrical optics formalism for the objective}

In order to achieve high geometrical magnification with a confined overall sample-to-detector distance, relatively short focal lengths are needed for the objective. This in turn implies that the CRL is composed of a large number of lenses, especially at hard X-ray energies. Thus, classical thin-lens formalisms are seldom adequate and more exact formalisms must be used instead (Simons et al., 2017). Here we recall the essential findings as relevant to dark-field microscopy. We shall assume that the CRL comprises $N$ identical paraboloid-shaped twodimensional lenses, each with a radius of curvature $R$, a distance between lenslet centres of $T$ and a web thickness between apices of $T_{\text {web }}$. Let $\delta$ and $\mu_{\text {att }}$ be the refractive decrement and the linear attenuation coefficient, respectively. The focal length corresponding to one lenslet then becomes $f=R /(2 \delta)$. It is shown that any ray passing through the objective will traverse a sinusoidal path. Let the ray emerge from a point in the sample plane at a radial distance $r_{\mathrm{s}}$ to the optical axis and at an angle of $\xi_{\mathrm{s}}$ to the direction of the optical axis. Then the sine curve is defined by a period of $2 \pi f \varphi$ and an amplitude of $\left[\left(r_{\mathrm{s}}+\xi_{\mathrm{s}} d_{1}\right)^{2}+\left(\xi_{\mathrm{s}} f \varphi\right)^{2}\right]^{1 / 2}$. Here $\varphi=(T / f)^{1 / 2}$.

With these definitions the focal length $f_{N}$ is

$$
f_{N}=f \varphi \cot (N \varphi) .
$$

The following relations apply between the magnification $\mathcal{M}_{\mathrm{CRL}}$ and the distances $d_{1}$ and $d_{2}$, defined in Fig. 1:

$$
d_{1}=f_{N}\left[1+\frac{1}{\mathcal{M}_{\mathrm{CRL}} \cos (N \varphi)}\right] ; \quad d_{2}=f_{N}\left[1+\frac{\mathcal{M}_{\mathrm{CRL}}}{\cos (N \varphi)}\right] .
$$

The transmission, $I / I_{0}$ (through the CRL), of a ray defined by $r_{\mathrm{s}}$ and $\xi_{\mathrm{s}}$ is

$$
I / I_{0}=\exp \left(-\mu_{\text {att }} N T_{\text {web }}\right) \exp \left[\frac{-\left(\xi_{\mathrm{s}}+\gamma r_{\mathrm{s}}\right)^{2}}{2 \sigma_{\mathrm{a}}^{2}}\right] \exp \left(\frac{-r_{\mathrm{s}}^{2}}{2 \sigma_{\mathrm{v}}^{2}}\right) \text {. }
$$

Here the three factors describe (i) attenuation in the centre 'web' part of the lenses, (ii) the pupil function leading to a Gaussian angular acceptance and thus numerical aperture with r.m.s. width $\sigma_{\mathrm{a}}$, and (iii) a Gaussian decay of intensity with distance from the detector centre (vignetting) with r.m.s. width $\sigma_{\mathrm{v}}$.

We have 


$$
\sigma_{\mathrm{a}}=\left(\frac{R}{N \mu_{\mathrm{att}}}\right)^{1 / 2} \frac{1}{d_{1}}\left[\left(1+S_{1}\right)+2 \frac{f \varphi}{d_{1}} S_{2}+\left(1-S_{1}\right) \frac{(f \varphi)^{2}}{d_{1}^{2}}\right]^{-1 / 2}
$$

with

$$
S_{1}=\operatorname{sinc}(2 N \varphi), \quad S_{2}=\frac{1}{N \varphi} \sin ^{2}(N \varphi) .
$$

For large $d_{1} / f \varphi$ this expression reduces to $\sigma_{\mathrm{a}}=\sigma_{\mathrm{D}} / d_{1}$, where $\sigma_{\mathrm{D}}$ is the spatial acceptance function for a parallel incident beam. To a good accuracy

$$
\sigma_{\mathrm{a}} \simeq \delta \frac{\mathcal{M}_{\mathrm{CRL}}}{\mathcal{M}_{\mathrm{CRL}}+1}\left(\frac{2 N}{\mu_{\mathrm{att}} R}\right)^{1 / 2} .
$$

The numerical aperture if defined by the FWHM is $\mathrm{NA}=2.35 \sigma_{\mathrm{a}}$. The last term in equation (6) defines the vignetting. We have

$$
\sigma_{\mathrm{v}}=\frac{2 \delta}{\mu_{\mathrm{att}} \sigma_{\mathrm{a}}}\left[(N \varphi)^{2}-\sin ^{2}(N \varphi)\right]^{-1 / 2} .
$$

For $N \rightarrow 0$ the expression diverges. Numerically we find it to be only approximately correct for $N<5$. For the characteristic distance $1 / \gamma$ in equation (6) we have

$$
\gamma^{2}=\left(\frac{\sigma_{\mathrm{a}}}{\sigma_{\mathrm{D}}}\right)^{2}-\left(\frac{\sigma_{\mathrm{a}}}{\sigma_{\mathrm{v}}}\right)^{2}
$$

It is shown by Simons et al. (2017) that the maximum $\sigma_{\mathrm{a}}$ for a given X-ray energy and type of lens is obtained in the limit of a maximally thick lens, where $N \varphi \simeq \pi / 2$ and the sampleobjective distance $d_{1}$ approaches 0 .

\section{Direct-space resolution}

When acquiring a three-dimensional map the spatial resolution may vary as a function of position (i.e. across the field of view). Employing the topo-tomography principle (Ludwig et al., 2001), the resolution related to a given region in the sample will also vary as function of the rotation angle $\omega$. In the absence of mechanical vibrations, small-angle scattering from the objective lens material and aberrations due to manufacturing errors, the prime contributions to the spatial resolution are the following:

The diffraction limit. When imaging 'on axis' (i.e. in the vicinity of the point on the detector that coincides with the optical axis), the angular acceptance function is defined by the r.m.s. value $\sigma_{\mathrm{a}}$ [equation (7)]. In classical optical systems, the resolution is often defined by the Rayleigh criterion (Lord Rayleigh, 1891), where two objects are considered resolved when they are further apart than the first dark ring of the point spread function (PSF) of the imaging system. Simons et al. (2017) argue that this is inappropriate in the case of a Gaussian or near-Gaussian PSF as is the case with CRLs. Instead, it is proposed that the resolution be defined by the separation distance between two PSFs corresponding to a contrast ratio of $C$ (where $C$ is small when the contrast is poor). In the case of absorption-limited (i.e. Gaussian) CRLs, this gives a function in terms of the wavelength $\lambda, \sigma_{\mathrm{a}}$ and $C$ :

$$
\Delta y_{\mathrm{d}}=[0.06905-0.1019 \log (1-C)]^{1 / 2}\left(\lambda / \sigma_{\mathrm{a}}\right) .
$$

The Rayleigh criterion corresponds to $C=0.26$; however, the value of $C$ necessary to distinguish two objects naturally depends on sampling statistics. In the case of low-intensity measurements, $C$ should be greater than 0.5 .

It follows from equation (6) that the width of the angular distribution off axis is the same as it is on axis. However, the intensities on the detector are weakened by a factor of $\exp \left[-r_{\mathrm{s}}^{2} /\left(2 \sigma_{\mathrm{v}}^{2}\right)\right]$ due to the vignetting. This change in intensity gives rise to a change in signal-to-noise ratio and hence affects contrast.

Magnification and signal-to-noise ratio. In the case of lownoise measurements, the combined magnification $\mathcal{M}=$ $\mathcal{M}_{\mathrm{CRL}} \mathcal{M}_{\mathrm{d}}$ of the X-rays in the $\mathrm{CRL}, \mathcal{M}_{\mathrm{CRL}}$, and in the detector system, $\mathcal{M}_{\mathrm{d}}$, implies a resolution of $d=d_{\mathrm{c}} /$ $\left(\mathcal{M}_{\mathrm{CRL}} \mathcal{M}_{\mathrm{d}}\right)$, where $d_{\mathrm{c}}$ is the spatial resolution of the camera itself. For simplicity, we shall set $d_{\mathrm{c}}=P$, where $P$ is the pixel size of the camera. In the common case in which a scintillator is used to convert the X-rays to visual light, a scintillator of optimal thickness will provide an X-ray-to-light conversion efficiency proportional to $\left(P / \mathcal{M}_{\mathrm{d}}\right)^{2}$ (Koch et al., 1998).

For an isotropically emitting source within the sample plane and on the optical axis, the intensity, $I$, registered in a given pixel of the detector is proportional to the product of the probing area in the sample system, the efficiency of the X-ray lens and the efficiency of the camera system:

$$
\begin{aligned}
I & \propto d^{2}\left[\sigma_{\mathrm{a}}^{2} \exp \left(-N \mu_{\mathrm{att}} T_{\mathrm{web}}\right)\right]\left(P / \mathcal{M}_{\mathrm{d}}\right)^{2} \\
& =d^{4} \sigma_{\mathrm{a}}^{2} \mathcal{M}_{\mathrm{CRL}}^{2} \exp \left(-N \mu_{\mathrm{att}} T_{\mathrm{web}}\right) .
\end{aligned}
$$

The signal-to-noise ratio $\mathrm{S} / \mathrm{N}$ therefore becomes

$$
\mathrm{S} / \mathrm{N} \propto d^{2} \sigma_{\mathrm{a}} \mathcal{M}_{\mathrm{CRL}} \exp \left(-N \mu_{\mathrm{att}} T_{\mathrm{web}} / 2\right) .
$$

As mentioned above, $\sigma_{\mathrm{a}}$ is optimal in the thick-lens limit, where for ideal lens manufacturing $\sigma_{\mathrm{a}} \rightarrow(2 \delta)^{1 / 2}$. For fixed spatial resolution $d$, this implies $\mathrm{S} / \mathrm{N} \propto \mathcal{M}_{\mathrm{CRL}}$. Generally speaking, it is favourable to achieve as much of the magnification as possible with the CRL rather than with the detector system, and preferably even using a direct detection system instead of an optically coupled scintillator. (In practice, very large values of $\mathcal{M}_{\mathrm{CRL}}$ are difficult to realize because of inaccuracies in lens manufacturing and minimum clearance requirements about the sample that impose a lower limit on the objective's focal length.)

Depth of field. According to classical optics, the depth of field $\sigma_{\text {depth }}$ is defined as the sum of a contribution from wave optics (the diffraction limit) and a contribution from geometric optics, defined by the resolution in the X-ray imaging plane, $y_{\mathrm{d}}$. The latter is readily evaluated using the raytransfer matrix (see e.g. Svelto, 2010) formalism. As a result

$$
2.35 \sigma_{\mathrm{depth}}=\frac{\lambda}{(\mathrm{NA})^{2}}+\frac{y_{\mathrm{d}}}{\mathcal{M}_{\mathrm{CRL}} \mathrm{NA}} \text {. }
$$


For $\mathrm{NA} \simeq 10^{-3}, \lambda \simeq 0.1 \mathrm{~nm}, \mathcal{M}_{\mathrm{CRL}} \simeq 10$ and $y_{\mathrm{d}} \simeq 1 \mu \mathrm{m}$, it appears that the two terms are of the same order of magnitude, and $\sigma_{\text {depth }} \simeq 100 \mu \mathrm{m}$, comparable to the field of view.

Chromatic aberration. Let $\varepsilon$ be defined as the relative X-ray energy, $\varepsilon=\Delta E / E$, of a ray impinging on the sample. Assume a Gaussian distribution in $\varepsilon$ with an r.m.s. width of $\sigma_{\mathrm{e}}$. Then the chromatic aberration gives rise to a point spread function that is Laplacian with the characteristic width $\sigma_{\mathrm{ch}}$ (Simons et al. (2017). ${ }^{1}$

$$
\sigma_{\mathrm{ch}}=\sigma_{\mathrm{a}} \sigma_{\mathrm{e}} d_{\mathrm{ch}} / \mathcal{M}_{\mathrm{CRL}}
$$

with the characteristic length

$$
\begin{aligned}
d_{\mathrm{ch}}= & N \varphi\left(\frac{d_{1} d_{2}}{f \varphi}-f \varphi\right) \cos (N \varphi) \\
& +\left[\frac{d_{1} d_{2}}{f \varphi}+f \varphi+N \varphi\left(d_{1}+d_{2}\right)\right] \sin (N \varphi) .
\end{aligned}
$$

Generally speaking for operation with single-crystal monochromators chromatic aberration is not an issue. For operation with pink beams see Falch et al. (2017).

\section{Reciprocal-space resolution}

\subsection{On axis, general case}

The angular resolution function has contributions from both the incident and diffracted beams. Here, we assume that both have a Gaussian profile with r.m.s. width $\Delta \zeta_{\mathrm{h}, \mathrm{v}}$ for the incident beam and $\sigma_{\mathrm{a}}$ for the objective lens's angular acceptance. Furthermore, we assume a normally distributed energy spread with r.m.s. width $\sigma_{\mathrm{e}}$. An additional contribution to the instrument resolution in the 'rocking' direction arises from the Darwin width of the sample material, which is somewhat complex to model (Als-Nielsen \& McMorrow, 2011). As it is of the order of $0.01-0.1 \mathrm{mrad}$ it is typically substantially smaller than the vertical divergence of the incoming beam, $\Delta \zeta_{\mathrm{v}}$, whenever a condenser is used. In the following we shall neglect the Darwin width.

In Appendix A4, equations for the reciprocal-space resolution are given for arbitrary motor positions. At the nominal working point $\eta=0,2 \theta=2 \theta_{\mathrm{B}}, \mu=\theta_{\mathrm{B}}$ the projections in the three directions in the reference system are found to be [equations (61)-(63)]

$$
\begin{gathered}
\Delta Q_{\text {rock }}=\frac{\left|\mathbf{Q}_{0}\right|}{2}\left(\Delta \zeta_{\mathrm{v}}^{2}+\sigma_{\mathrm{a}}^{2}\right)^{1 / 2}, \\
\Delta Q_{\text {roll }}=\frac{\left|\mathbf{Q}_{0}\right|}{2 \sin \left(\theta_{\mathrm{B}}\right)}\left(\Delta \zeta_{\mathrm{h}}^{2}+\sigma_{\mathrm{a}}^{2}\right)^{1 / 2}, \\
\Delta Q_{\|}=\frac{\left|\mathbf{Q}_{0}\right|}{2}\left[\left(2 \sigma_{\mathrm{e}}\right)^{2}+\cot ^{2}\left(\theta_{\mathrm{B}}\right)\left(\Delta \zeta_{\mathrm{v}}^{2}+\sigma_{\mathrm{a}}^{2}\right)\right]^{1 / 2} .
\end{gathered}
$$

This result can be misleading, as the principal axes of the resolution function are not parallel to the axes of the coordinate system defined. In fact, it is often useful instead to operate in the imaging coordinate system. A basic Monte

\footnotetext{
$\overline{1}$ There is an error in this equation in the work of Simons et al. (2017).
}

Carlo program has been generated to provide three-dimensional plots based on statistical distributions of rays and applying equations (19), (20) and (21). An example result for a case with large incoming divergence is shown in Fig. 4(a) below (\$5.1).

Inspection of equations (19)-(21) reveals that the horizontal divergence/acceptance affects only the 'roll' component of the resolution, and the bandwidth only the longitudinal resolution. The vertical divergence/acceptance affects both the longitudinal and 'rock' components of the resolution. Furthermore, it is evident that the relative longitudinal resolution, $\Delta Q_{\|} /\left|\mathbf{Q}_{0}\right|$, diverges for $\theta_{\mathrm{B}} \rightarrow 0$ (forward scattering) as $\left|\mathbf{Q}_{0}\right| \rightarrow 0$. It continuously improves with increasing $\theta_{\mathrm{B}}$. At $2 \theta_{\mathrm{B}} \rightarrow 180^{\circ}$, i.e. back scattering, the longitudinal resolution is determined only by $\sigma_{\mathrm{e}}$.

4.2. On axis, case of large asymmetry between incoming and diffracted beam

In the following we describe a setup where the incoming beam is defined by a standard double-crystal monochromator with $\sigma_{\mathrm{e}}$ of order $10^{-4}$ and exhibits a small divergence of $\Delta \zeta_{\mathrm{h}, \mathrm{v}} \simeq 0.1 \mathrm{mrad}$ or less. In comparison the divergence of the diffracted beam is much larger: $\sigma_{\mathrm{a}} \simeq 1 \mathrm{mrad}$. In this case the resolution function becomes a three-dimensional Gaussian with axes parallel to the axes of the imaging coordinate system. As derived in Appendix $A$ [equations (56), (70) and (71) using $\Delta \xi_{\perp, \|}=\sigma_{\mathrm{a}}$,

$$
\begin{gathered}
\Delta Q_{\text {rock }^{\prime}} \simeq \frac{\left|\mathbf{Q}_{0}\right|}{2} \cos \left(\theta_{\mathrm{B}}\right) \Delta \zeta_{\mathrm{v}}, \\
\Delta Q_{\mathrm{roll}} \simeq \frac{\left|\mathbf{Q}_{0}\right|}{2 \sin \left(\theta_{\mathrm{B}}\right)} \sigma_{\mathrm{a}}, \\
\Delta Q_{2 \theta} \simeq \frac{\left|\mathbf{Q}_{0}\right|}{2 \tan \left(\theta_{\mathrm{B}}\right)} \sigma_{\mathrm{a}} .
\end{gathered}
$$

It appears that the resolution function is very anisotropic:

$$
\Delta Q_{\text {rock' }^{\prime}} \ll \Delta Q_{\text {roll }} \simeq \Delta Q_{2 \theta} .
$$

In this case, to a good accuracy the resolution function can be approximated by a circular disc. A Monte Carlo simulation with the same parameters confirms this ( $c f$. Fig. $4 b$ below).

For very large anisotropies, as defined by equation (25), second-order effects become important. The tangential plane approximation for the Ewald sphere is valid for the achievable numerical apertures, but the fact that the resolution function lives in the imaging system while a rotation of $\chi$ takes place in the reference system has implications. For example, imagine scanning a perfect crystal in $\chi$ as an approximation to determine the resolution in the rolling direction, as defined by $\Delta \eta$. As derived in Appendix $A$, for $\omega=0$ the angular distance between $\mathbf{Q}$ and the Ewald sphere is

$$
\Delta \phi=\left[1-\cos \left(\theta_{\mathrm{B}}\right)\right]\left|\chi-\chi_{0}\right| .
$$



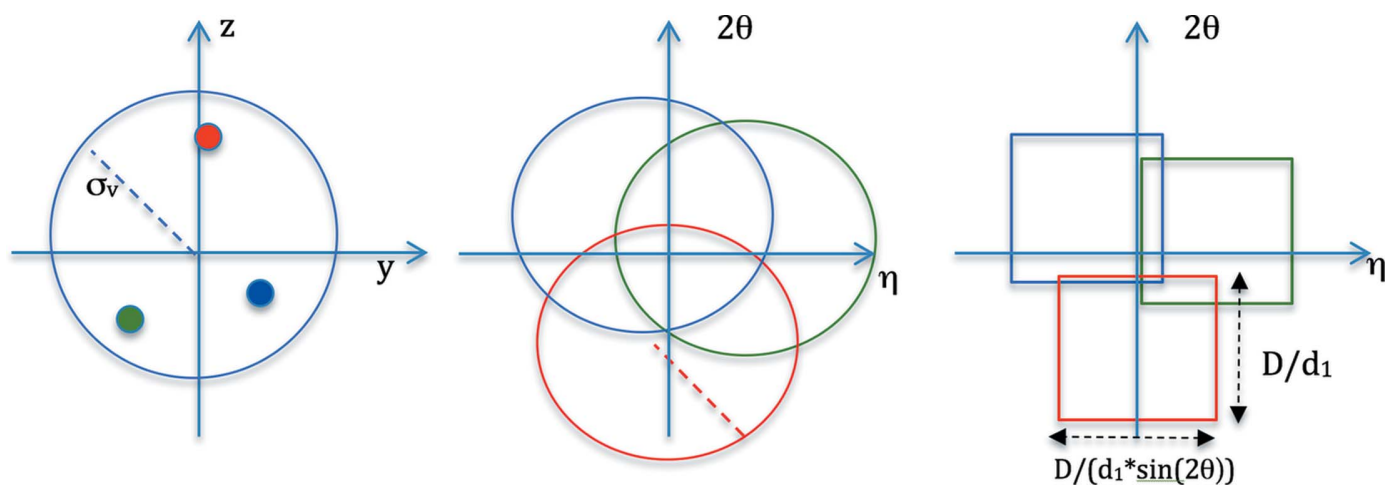

Figure 3

Coupling between direct and reciprocal space. Left: the $(y, z)$ sample plane with a circle marking the cutoff by vignetting and three randomly selected points in this plane, identified by colour. Middle: the $(\eta, 2 \theta)$ plane with circles marking the angular acceptance of the objective [cf. equation (7)]. The length of the dashed red line is $\sigma_{\mathrm{a}}$ in direction $2 \theta$ and $\sigma_{\mathrm{a}} / \sin (2 \theta)$ in direction $\eta$. The three circles are seen to be offset according to the ( $\left.y, z\right)$ position identified by the same colour. Right: a similar plot for the case of having inserted a square aperture at the entry point of the lens. The aperture size is $D$ and $D / d_{1} \leq \sigma_{\mathrm{a}} / 2^{1 / 2}$.

For large anisotropies this means that the scattering vector 'falls off the rocking curve' before it has traversed the full range $\Delta \eta$.

\subsection{Off axis}

When moving off axis $\left(r_{\mathrm{s}} \neq 0\right)$ the angular distribution, as defined by the middle term in equation (6), changes angular position but its width is maintained. This implies that the reciprocal-space resolution distributions in the radial and azimuthal directions have the same shapes when moving off the optical axis, but the centre of the distribution is shifted. For a pixel on the two-dimensional detector corresponding to a position $\left(y_{\mathrm{s}}, z_{\mathrm{s}}\right)$ within the sample plane, we have the following shifts:

$$
\begin{gathered}
\eta_{\text {shift }}=\frac{\gamma y_{\mathrm{s}}}{2 \sin (\theta)} ; \Delta Q_{\text {shift,roll }}=-\frac{\left|\mathbf{Q}_{0}\right| \gamma y_{\mathrm{s}}}{2 \sin (\theta)} ; \\
2 \theta_{\text {shift }}=-\gamma z_{\mathrm{s}} ; Q_{\text {shift }, \|}=-\frac{\left|\mathbf{Q}_{0}\right| \gamma z_{\mathrm{s}}}{2 \tan (\theta)} ; \Delta Q_{\text {shift,rock }}=\frac{\left|\mathbf{Q}_{0}\right| \gamma z_{\mathrm{s}}}{2 \tan (\theta)} .
\end{gathered}
$$

This coupling between direct- and angular-space resolution is illustrated in Fig. 3.

\section{Sampling}

\subsection{Optimizing the reciprocal-space resolution function}

Sampling is a major concern in both direct and reciprocal space: objects of interest may be larger than the field of view, and reflections tend to be broader than the NA. The inherent coupling between two direct- and two reciprocal-space coordinates (illustrated in Fig. 3) combined with a complicated resolution function complicates this issue. Sampling is also a concern when comparing images acquired at different magnifications, as vignetting and reciprocal-space resolution change as functions of lens parameters. Furthermore, when combining data from different positions of $\omega$ it will typically be necessary to operate in the entire six-dimensional $(x, y, z, \phi, \chi, 2 \theta)$ space for reasons of sampling.

An additional major sampling issue is that one cannot make a space-filling disjunct map in two dimensions by placing circles next to each other, as shown in Fig. 3. Hence, when scanning in $\chi$ or $2 \theta$, parts of the distribution may be sampled twice and other parts not at all. This is a particular concern in connection with the use of tomography-type reconstruction algorithms, as these are based on a linear relationship between diffracting volume and integrated intensity (Kak \& Slaney, 1988).

In the following, we propose a strategy for enabling mapping which is space filling in reciprocal space. Essentially, this involves making the reciprocal-space resolution box shaped. Specifically, we consider the case of large anisotropy, presented in $\S 4.2$. First, we propose to insert a square aperture in front of the CRL. This implies that the resolution function in the $(\eta, 2 \theta)$ plane becomes a rectangular box multiplied with the Gaussian angular acceptance function. This is illustrated in Fig. 3(c), for comparison with Fig. 3(b), and in Fig. 4(c), for comparison with Fig. 4(b).

Next, to change the width in the rocking direction, one may integrate the signal while rotating with a constant speed within a given angular range, $W_{\phi}$. The result of such a 'sweep' becomes a uniform sampling in direction $\phi$ and the corresponding resolution function is approximately a box function. As a result the overall reciprocal-space resolution function has become box shaped in all three directions in the imaging coordinate system, as shown in Fig. $4(d)$.

The resulting FWHM widths become

$$
\begin{gathered}
\Delta Q_{\text {rock }^{\prime}}=\left|\mathbf{Q}_{0}\right| W_{\phi}, \\
\Delta Q_{\text {roll }}=\left|\mathbf{Q}_{0}\right| \frac{D}{\sin (2 \theta) d_{1}}, \\
\Delta Q_{2 \theta}=\left|\mathbf{Q}_{0}\right| \frac{D}{2 \tan \left(\theta_{\mathrm{B}}\right) d_{1}},
\end{gathered}
$$

where $D$ is the size of the opening in the slit. 

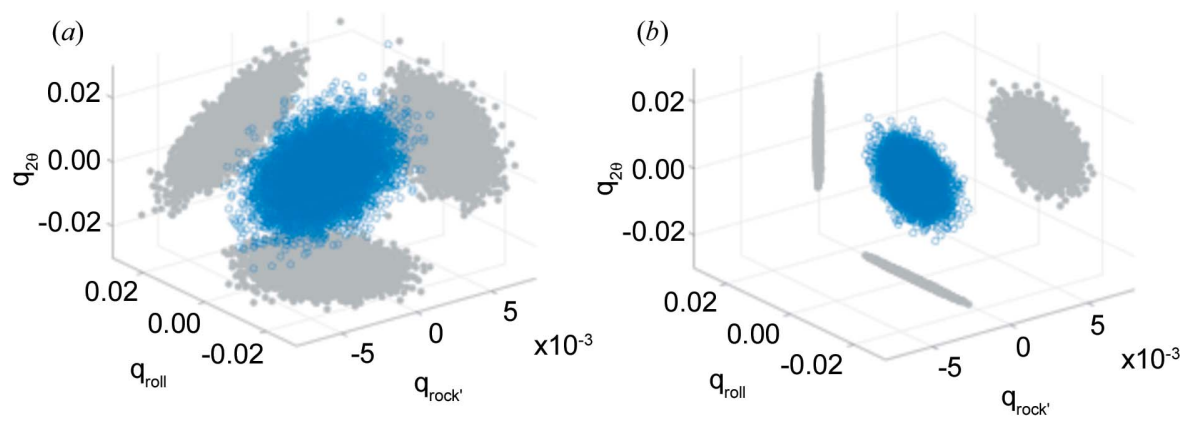

(c)

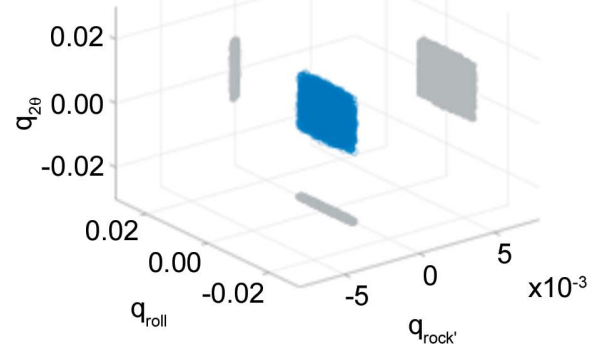

Figure 4

Examples of reciprocal-space resolution functions, as described in the imaging system, with shadow plots. All simulations are performed with $\left|\mathbf{Q}_{0}\right|=31.1 \mathrm{~nm}^{-1}, \theta=10.39^{\circ}, \sigma_{\mathrm{a}}=0.5 \mathrm{mrad}$ and $\sigma_{\mathrm{e}}=6 \times$ $10^{-5}$. (a) Case of condenser with similar setting: $\zeta_{\mathrm{v}}=\zeta_{\mathrm{h}}=\sigma_{\mathrm{a}}$. (b) Case of low incoming divergence: $\zeta_{\mathrm{v}}=\zeta_{\mathrm{h}}=10^{-5}$. (c) As in (b) but with a slit in front of the CRL with an opening of 1 mrad. (d) As in (c) but with continuous scanning in $\phi$ during exposure within a range of 1 mrad.

$$
\Delta \mu=\frac{\Delta Q_{\text {rock'}^{\prime}}}{\left|\mathbf{Q}_{0}\right|}
$$

Axial strain mapping. When mapping the axial strain, an intrinsic strain resolution of $10^{-4}$ or better may be required. High-resolution mapping of the strain distribution for each voxel in the sample is therefore excluded, but it is still possible to derive the average value for the axial strain component for each voxel. Similar to the case of neutron strain scanning using a monochromatic beam (Hutchings et al., 2005), we can exploit the symmetry and our a priori knowledge of the resolution function, $c f$. $\S$. With sufficient count rates, the centre position can be found to an accuracy that can be as good as $1 \%$ of the width. The neutron community has provided algorithms for reducing the number of scanning points required and optimizing $\mathrm{S} / \mathrm{N}$. We find these results to be applicable for dark-field microscopy as well.

To reduce the dimension it is often

Mapping of larger regions of reciprocal space can now be obtained in a space-filling way by sampling disjunct cubeshaped 'voxels' in reciprocal space. The added aperture will, however, reduce the NA and therefore lead to deterioration of the spatial resolution.

\subsection{Sampling strategies}

Three-dimensional sampling of reciprocal space is time consuming and, in practice, two projections of reciprocal space have proven of particular use:

Mosaicity maps. With a numerical aperture of order $\sigma_{\mathrm{a}}=1 \mathrm{mrad}$ and scattering angles of order $2 \theta=25^{\circ}$, the intrinsic strain resolution becomes $\Delta Q /\left|\mathbf{Q}_{0}\right|=\cot (\theta) \Delta \theta \simeq$ $2.2 \times 10^{-3}$ (r.m.s. value). For many specimens, this value is sufficiently large that one can integrate over the entire axial strain distribution. [For low magnifications this scheme may not be valid at the top and bottom of the detector, owing to the offsets, $c f$. equations (27) and (28).] In this case a mosaicity map can be provided by a regular two-dimensional scan, with the first movement being a linear combination of $\phi$ and $\chi$ and the second a 'rock' in base tilt $\mu$. The step sizes are

$$
\begin{aligned}
& \Delta \phi=\frac{\sin (\omega)}{\cos \left(\chi_{0}\right)} \frac{\Delta Q_{\text {roll }}}{\left|\mathbf{Q}_{0}\right|}, \\
& \Delta \chi=\cos (\omega) \frac{\Delta Q_{\text {roll }}}{\left|\mathbf{Q}_{0}\right|},
\end{aligned}
$$

relevant to scan $\chi$ and $2 \theta$ (for $\omega=0$ ) in a two-dimensional grid, while for each point integrating over the entire rocking curve by a continuous scan in $\phi$ or $\mu$. During data analysis the bins in $\chi$ are summed to provide a single axial strain value for each voxel in the sample.

As an alternative, mapping of mosaicity and axial strain can be performed by scanning of an aperture in the back focal plane of the objective. Imaging and operations in the back focal plane are outside the scope of this article and will be the topic of an upcoming publication.

\section{Experimental demonstration}

To demonstrate the correspondence of the formalism with empirical data, we report on experimental results obtained from a diamond single crystal. Using an Si(111) Bragg-Bragg double-crystal monochromator, the energy was fixed at $17 \mathrm{keV}$ with $\sigma_{\mathrm{e}} \simeq 6 \times 10^{-5}$. The diamond crystal was cut with a (11̄0) surface normal and mechanically polished to $420 \mu \mathrm{m}$ thickness. Carrying out dark-field X-ray microscopy on a 111 reflection in symmetric Laue geometry, the scattering angle is $2 \theta_{\mathrm{B}}=20.317^{\circ}$. An absorption test grid was placed in the diffracted beam in close proximity to the diamond crystal. This comprised a pattern of $\sim 1 \mu \mathrm{m}$ thick $\mathrm{W}$ lines, corresponding to an increased absorption of $15 \%$.

The incident beam was shaped by an up-stream condenser, leading to a near-Gaussian rocking curve with a width of $0.029 \mathrm{mrad}$ (FWHM) (see Fig. 5, left). For comparison, a Gaussian curve with a width defined by the Darwin curve for 
this reflection is also shown. The test was made with a uniform incident beam illuminating an area larger than the field of view of the microscope and an exposure time of $1 \mathrm{~s}$. The objective comprised $N=69$ identical Be lenslets with a radius of $R=50 \mu \mathrm{m}$ and thickness of $T=1.6 \mathrm{~mm}$. Hence $2 \delta=2.359 \times 10^{-6}, \mu_{\text {att }}=47 \mathrm{~m}^{-1}, f=21.195 \mathrm{~m}, \varphi=0.00869$ and $f_{\mathrm{N}}=0.269 \mathrm{~m}$. The focus position was experimentally optimized at $d_{1}=0.289 \pm 0.002 \mathrm{~m}$ with a magnification of $\mathcal{M}_{\mathrm{CRL}}=15.1$ which, within experimental error, corresponds to the predicted value. From this follows a prediction for $\mathrm{NA}(\mathrm{FWHM})=2.35 \sigma_{\mathrm{a}}=0.63 \mathrm{mrad}$.
Fig. 5 (right) shows the intensity variation at the centre of the detector, corresponding to the on-axis position for a $\chi$ scan. To improve $\mathrm{S} / \mathrm{N}$, the signal was averaged over a region of interest $(\mathrm{ROI})$ with $40 \times 40$ pixels. The curve has a width of $1.05 \mathrm{mrad}$. This is compared with two theoretical predictions: the blue line is a direct comparison with the predicted value for $\Delta Q_{\text {roll }}, c f$. equation (23); the red line takes into consideration the fact that the scattering vector falls off the rocking curve, $c f$. equation (26).

The $2 \theta$ resolution was measured by scanning the $2 \theta$ arm using a combined movement of the objective and the detector.
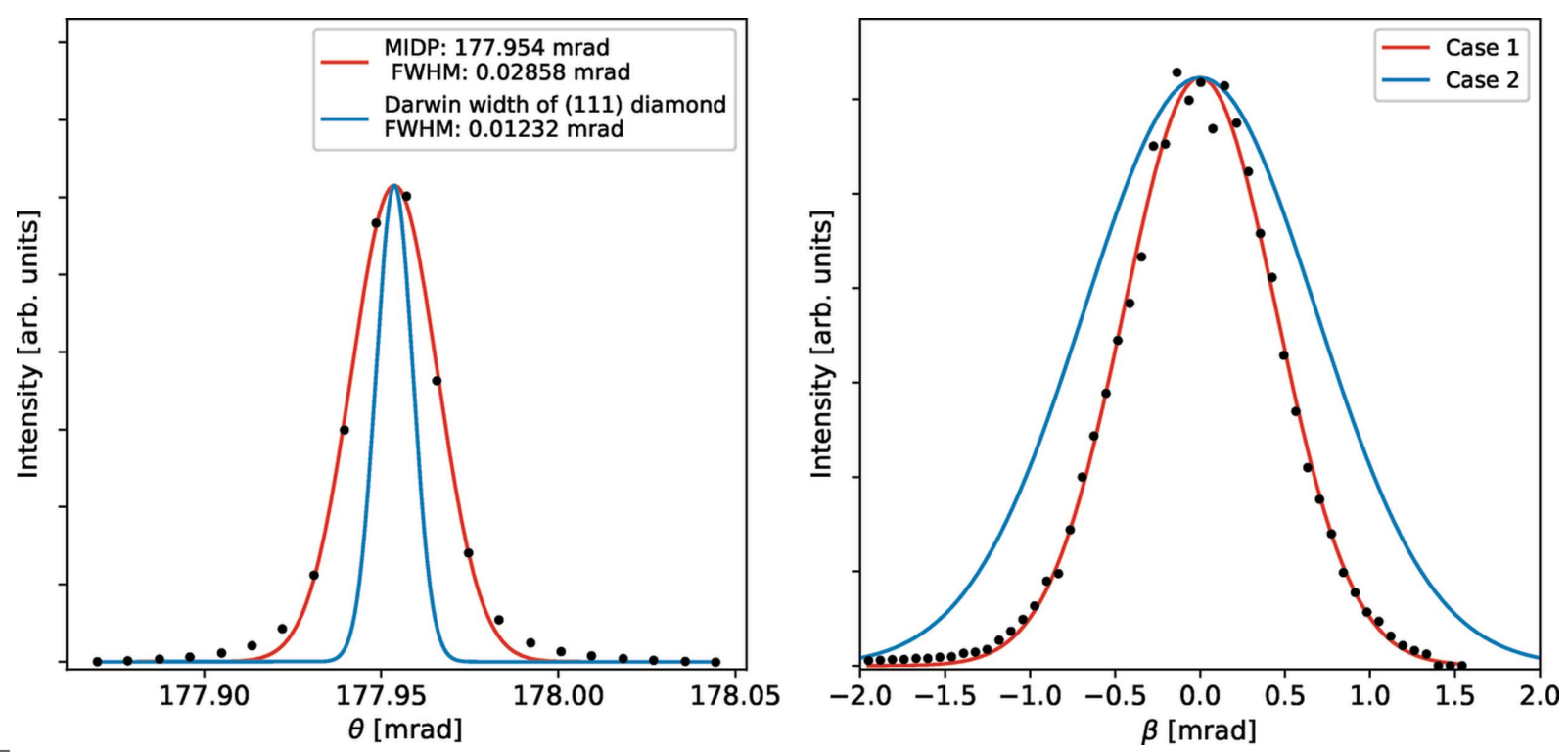

Figure 5

Experimental tests of the angular resolution. Left: raw data (dots) and a fit to a Gaussian (full line) for a rocking curve, that is a scan in $\phi$ as defined in Fig. 1. Right: raw data (dots) for a scan along the rolling direction $\chi$. Also shown as full lines are two predictions - see main text.
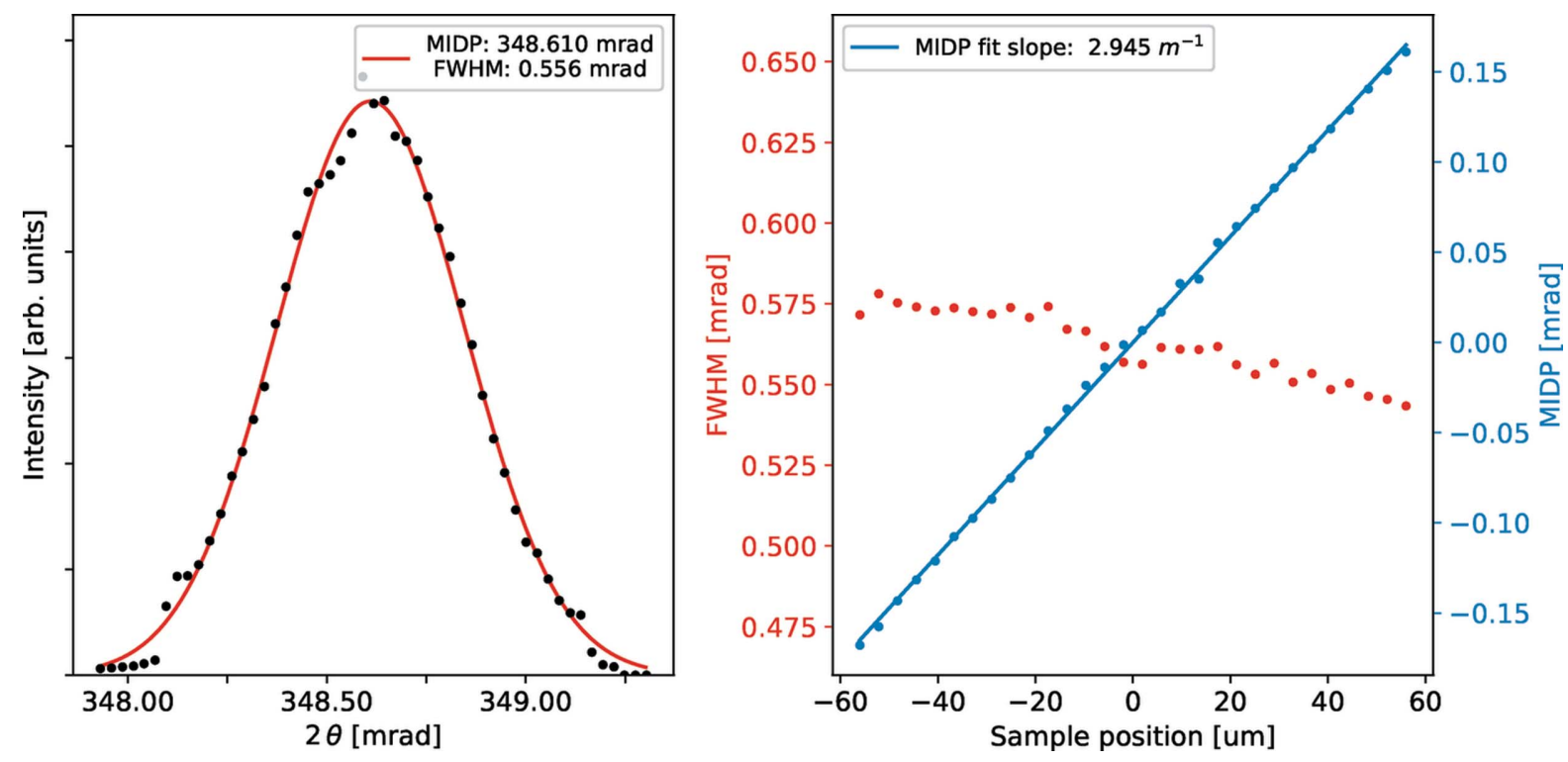

Figure 6

Experimental test of $2 \theta$ resolution. Left: raw data (dashed line) and a fit to a Gaussian (full line) for the on-axis case. Right: the FWHM (red dots) and the offset in midpoint (blue dots) resulting from a set of Gaussian fits to $2 \theta$ resolution curves - similar to that to the left - as a function of how far off axis the sample position is. The blue line represents a fit of the blue dots to a straight line. 
For various positions on the detector, the intensity variation with $2 \theta$ was recorded. To improve $\mathrm{S} / \mathrm{N}$, again, the signal was averaged over an ROI with $40 \times 40$ pixels. The result for the centre of the detector (corresponding to the on-axis position) is shown in Fig. 6 (left). The curve is well described by a Gaussian with a width (FWHM) of $0.56 \mathrm{mrad}$. This should be compared with the predicted value of NA $=0.63 \mathrm{mrad}$. The discrepancy is attributed to errors in alignment and lens manufacture.

The $2 \theta$ intensity distribution remained Gaussian when offsetting the centre of the ROI in the vertical direction. Fig. 6 (right) shows the resulting centre positions and FWHM values from Gaussian fits to these profiles. As predicted by the formalism above, the width remains constant, while there is a linear dependence between vertical offset within the sample and the offset in $2 \theta$ [cf. equation (27)]. The slope fitted to the experimental data, $\gamma=2.94 \mathrm{~m}^{-1}$, agrees well with the predicted value, $\gamma=2.93 \mathrm{~m}^{-1}$.

\section{Discussion}

Crystalline materials are characteristically organized into grains and domains in a hierarchical fashion. Mapping all domains in a sample with say 1000 grains requires a Herculean effort. Within a multiscale approach, we propose instead initially to map the entire sample on a coarse scale using 3DXRD, DCT or similar. Having identified a grain of interest we then zoom in on this and provide a fine map of the domains with dark-field microscopy. Similar to the operation of a transmission electron microscope (Williams \& Carter, 2009; Midgley \& Eggemann, 2015), we find it advantageous to combine dark- and bright-field X-ray microscopy in one setup, for ease of alignment purposes and to combine diffractionbased microscopy with local phase contrast mapping (e.g. Falch et al., 2017) and with very fast three-dimensional mapping of phases, voids, cracks etc. (Falch et al., 2017).

The six-dimensional resolution analysis presented here is based on the geometrical optics treatment of CRLs by Simons et al. (2017). This implies that certain aspects of diffraction and refraction have not been taken into account - for this we refer to more elaborate methods (e.g. Kohn, 2002; Knudsen et al., 2013). However, we have verified by direct comparison with wavefield simulations that the presented analytical expressions, for example, for the numerical aperture are accurate within experimental error (for typical configurations of the CRL) and they can easily be implemented in a numerical optimization of the experimental setup.

It has been shown that CRLs can, in principle, provide a spatial resolution of $10 \mathrm{~nm}$ or below (Schroer \& Lengeler, 2005). In practice, however, the resolution is limited in two ways. Firstly, the numerical aperture for one refractive optics element is fundamentally limited to $(2 \delta)^{1 / 2}$. Secondly, and more importantly, so far the various manufacturing routes (Lengeler et al., 1999; Schroer \& Lengeler, 2005; Krywka et al., 2016; Simons, Stöhr et al., 2016) have all been associated with aberrations and other optical imperfections that limit the resolution to approximately $100 \mathrm{~nm}$. Diffractive optics have the potential to overcome both of these limitations. At energies below $15 \mathrm{keV}$, bright-field $\mathrm{X}$-ray microscopes using Fresnel zone plates exhibit a spatial resolution of the order of tens of nanometres (Vila-Comamala et al., 2012). At higher energies, multilayer Laue lenses have recently been demonstrated (as condensers) with an NA of 0.006 at $\lambda=0.056 \mathrm{~nm}$ (Morgan et al., 2015), with potential for further improvement.

The angular resolution of dark-field X-ray microscopy is superior to that of a transmission electron microscope when operated for typical materials science inspection. The high angular resolution has two advantages:

Firstly, one fundamental limitation to any nondestructive three-dimensional diffraction technique is the overlap of diffraction signals. Illuminating a $1 \mathrm{~mm}$ thick sample comprising domains with a volume of $(1 \mu \mathrm{m})^{3}$ with a beam of $100 \times 100 \mu \mathrm{m}$ implies that the beam simultaneously interacts with $10^{7}$ domains. In comparison, grain mapping methods like 3DXRD are limited to around 3000 simultaneously illuminated grains (Sørensen et al., 2012). With dark-field microscopy, domains diffracting in directions outside the solid angle defined by the NA on the unit sphere are invisible. This solid angle is $\xi=\mathrm{NA} \Delta \zeta /(4 \pi)$. Let $M$ be the multiplicity of the relevant reflection(s) within the $2 \theta$ range covered. Then the likelihood of observing a randomly oriented domain is $p=\xi M$. For the ID06 setup mentioned above and $M=10, p$ is of order $10^{-8}$. Furthermore, in this case the field of view of the microscope will correspond to $1 / 10$ of the thickness of the sample and the number of pixels on the detector is $10^{6}$. It appears that, for a random distribution of orientations of the domains, the likelihood of spot overlap is negligible. For even smaller domains $(100 \mathrm{~nm})$, it appears one may be able to isolate one domain out of a billion.

Secondly, when rotating about the scattering vector, $\mathbf{Q}$, the strongly asymmetric $(\phi, \chi)$ resolution function also rotates. Given the formalism provided in this paper, one may attempt to construct super-resolution algorithms (e.g. Richter et al., 2016) aiming for an angular resolution close to the Darwin width in both directions. This would provide unprecedented data for modelling local disorder, e.g. dislocation densities.

On the other hand, for grains and domains exhibiting a large degree of mosaic spread, the small NA of CRLs implies that the generation of a reciprocal-space-filling map becomes tedious. For such cases again diffractive optics are an interesting alternative.

It should be emphasized that the approach outlined above only probes reciprocal space in the vicinity of one scattering vector. Hence, the full orientation of the domains is not determined, and only three out of the nine components of the displacement gradient tensor are monitored. To provide a full description, the mapping has to be repeated for at least two other non-collinear reflections associated with the same domain. This is currently not possible without re-mounting the sample, owing to the restricted travel of the $\chi$ and $\phi$ axes.

In outlook, we remark that the product of direct-space and reciprocal-space resolution is proportional to $\lambda$ for both refractive and diffractive optics. Very high energy X-rays may therefore be a route forward for future six-dimensional 
microscopy approaches, combining modelling in both direct and reciprocal space. We also remark that the concept of a dark-field neutron microscope and its implementation in a time-of-flight operation was proposed by Poulsen et al. (2014). Similar to the X-ray case, dark-field neutron microscopy may be seen as part of a multi-scale approach complementary to neutron absorption tomography and neutron diffraction tomography of grains (Peetermans et al., 2014; Cereser et al., 2017).

\section{Conclusion}

A comprehensive formalism has been established for operating a dark-field X-ray microscope. With variation of the wavelength, focal distance, magnification and range of continuous scans, the microscope provides ample possibilities for optimizing spatial, angular and time resolution, field of view etc. For a small incoming beam divergence, the reciprocal space resolution element is a very anisotropic platelet with a surface normal along the optical axis of the diffracted beam. In order to make a disjunct space-filling sampling, the resolution function should be approximately a box function in all directions. This can be achieved by inserting a square slit in front of the CRL and by sweeping in $q_{\text {rock }}$. By a sweep of a sufficiently large range, the anisotropy can be removed. A composite map of a larger fraction of reciprocal space can be obtained by scanning in $(\chi, \phi, 2 \theta)$ while conserving integrated intensity. For voxels away from the optical axis additional shifts apply [cf. equations (27) and (28)], as one is probing different regions of reciprocal space when varying positions $y$ and $z$ in the sample at fixed angles $\phi, 2 \theta$. Potentially there are also changes in intensity due to vignetting.

\section{APPENDIX $A$}

\section{Angle calculations for the HXRM instrument}

The purpose of this appendix is to derive all angle and resolution calculations in the most general setting. These are then considered for the simplified case of nominal operating conditions as discussed in the main paper.

We start with the definition of the scattering geometry, followed by the treatment of the resolution in reciprocal space due to the divergence and energy bandwidth of the incident beam and the objective lens's angular acceptance. Next, we consider the sample rotations and the displacements in reciprocal space that small angular movements correspond to these are related to the step size in scans designed to cover reciprocal space in a disjunct and space-filling fashion.

These calculations do not take into account the imaging geometry of the experiment. Instead, we consider only geometrical vectors, $\mathbf{k}_{\text {in }}$ and $\mathbf{k}_{\text {out }}$, through the centre of rotation of the goniometer and along the optical axis of the objective lens.

The imaging geometry, where points of interest are not located on the optical axis but away from the instrument's centre of rotation, will be discussed in the following section.
The geometry of the dark-field X-ray microscope is sketched in Fig. 7. The geometry has been inspired by existing 3DXRD systems (Poulsen et al., 2001; Hefferan et al., 2012; Poulsen, 2012; Schmidt, 2014), as we aim to perform 3DXRD for initial sample characterization on the same instrument, and to directly import orientation matrices from 3DXRD data analysis into the dark-field X-ray microscopy experiment.

The sample is mounted on top of four rotation stages as shown in Fig. 7. As reference we utilize the laboratory coordinate system, with the $x$ axis along the incident beam and the $z$ axis vertical. From bottom, i.e. the laboratory coordinate system, to top, the sample goniometer axes are the 'base tilt' $\mu$ about $y$ (left handed), the 'sample azimuth' $\omega$ about $z$, and the 'sample tilts' $\chi$ about $x$ and $\phi$ about $y$ (left handed).

The detector moves in two directions such that the incident and diffracted beams form the scattering angle $2 \theta$ (see Fig. 7). Additionally, the detector can be rotated about the incident beam ('detector azimuth' $\eta$ ) to allow for the observation of oblique reflections, for example when the sample orientation is pre-defined by sample environments such as load frames for stress-strain studies or electric fields for ferroelectrics.

The convention for $\eta$ to rotate about the incident beam was chosen for compatibility with 3DXRD (Poulsen et al., 2001; Hefferan et al., 2012; Poulsen, 2012; Schmidt, 2014).

Compared to 3DXRD, we introduce additional sample rotations. In 3DXRD, the base tilt $\mu$ and the sample tilts $\chi$ and $\phi$ are generally assumed to be zero, such that the only remaining axis, $\omega$, is perpendicular to the incident beam.

For topo-tomo-type experiments (Ludwig et al., 2001) it is necessary to rotate the sample about the scattering vector. For this, as we will see below, it is most convenient to operate with a vertical scattering plane, i.e. $\eta=0$, and in bisecting geometry, $\mu=\theta$. We refer to this as the 'nominal working point'. In this case $\omega$ rotates about the scattering vector, i.e. the diffraction condition is fulfilled independently of $\omega$.

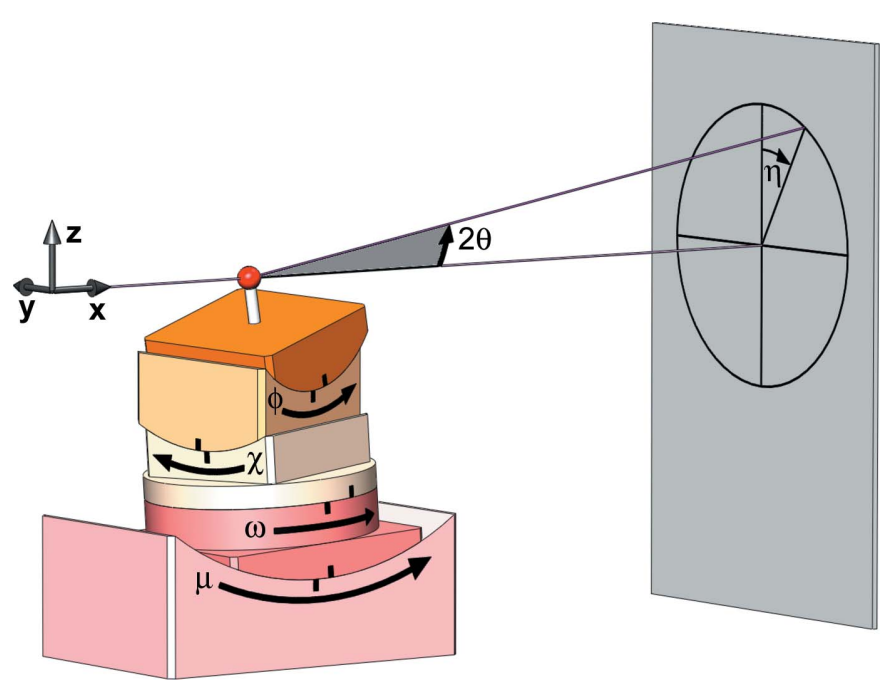

Figure 7

Geometry of the HXRM instrument. All rotations are shown at a positive angle. Note that the $\mu$ and $\phi$ rotations are left handed, whereas all other rotations are right handed. The origin of the laboratory coordinate system is located at the centre of rotation of the goniometer. 
The $\chi$ and $\phi$ stages are implemented as circle segments with moderate travel, $|\chi|,|\phi|<10^{\circ}$.

The resulting configuration is thus very similar to the ' $4 \mathrm{~S}+$ 2D' six-circle diffractometer (sometimes referred to as 'PSIC' diffractometer) described by You (1999). Note, however, the different configuration of the detector axes. At $\omega=\eta=0$ the geometry is identical to the four-circle diffractometer described by Busing \& Levy (1967).

The different rotation stages of the instrument are treated in the usual way (Busing \& Levy, 1967; Vlieg, 1997; You, 1999) as transformations between a series of coordinate systems. Each rotation stage is represented by a rotation matrix.

\section{A1. Laboratory coordinate system and incident beam}

The laboratory coordinate system is chosen with $x$ along the direction of the incident X-ray beam, $z$ vertical and $y$ horizontally to port (see Fig. 7). Vectors in the laboratory coordinate system are marked with the subscript 'lab'.

The incident beam vector is thus given by

$$
\mathbf{k}_{\text {in }}=k \hat{x}_{\text {lab }},
$$

where $k=2 \pi / \lambda$ and $\lambda$ is the X-ray wavelength.

The bandwidth of the beam and its divergence will be treated below.

\section{A2. Detector}

The detector moves in two directions, $2 \theta$ and $\eta$, as shown in Fig. 7.

Let $\mathbf{R}_{x}(\alpha), \mathbf{R}_{y}(\alpha)$ and $\mathbf{R}_{z}(\alpha)$ be right-handed rotation matrices about the $x, y$ and $z$ axes, respectively:

$$
\begin{aligned}
& \mathbf{R}_{x}(\alpha)=\left[\begin{array}{ccc}
1 & 0 & 0 \\
0 & \cos (\alpha) & -\sin (\alpha) \\
0 & \sin (\alpha) & \cos (\alpha)
\end{array}\right], \\
& \mathbf{R}_{y}(\alpha)=\left[\begin{array}{ccc}
\cos (\alpha) & 0 & \sin (\alpha) \\
0 & 1 & 0 \\
-\sin (\alpha) & 0 & \cos (\alpha)
\end{array}\right], \\
& \mathbf{R}_{z}(\alpha)=\left[\begin{array}{ccc}
\cos (\alpha) & -\sin (\alpha) & 0 \\
\sin (\alpha) & \cos (\alpha) & 0 \\
0 & 0 & 1
\end{array}\right] .
\end{aligned}
$$

In the laboratory coordinate system, the diffracted beam is then given by

$$
\mathbf{k}_{\mathrm{out}}=k \mathbf{R}_{x}(\eta) \mathbf{R}_{y}(-2 \theta) \hat{x}_{\mathrm{lab}}=k\left[\begin{array}{c}
\cos (2 \theta) \\
-\sin (2 \theta) \sin (\eta) \\
\sin (2 \theta) \cos (\eta)
\end{array}\right]_{\mathrm{lab}} .
$$

This in turn gives the scattering vector as

$$
\mathbf{Q}=\mathbf{k}_{\text {out }}-\mathbf{k}_{\text {in }}=k\left[\begin{array}{c}
\cos (2 \theta)-1 \\
-\sin (2 \theta) \sin (\eta) \\
\sin (2 \theta) \cos (\eta)
\end{array}\right]_{\text {lab }},
$$

with $|\mathbf{Q}|=2 k \sin (\theta)$ for all values of $\eta$.

The Bragg condition, $|\mathbf{Q}|=2 k \sin \left(\theta_{\mathrm{B}}\right)$, thus determines $\theta=\theta_{\mathrm{B}}$. Furthermore, the instrument generally operates in the vertical scattering plane such that $\eta=0$. These two conditions fully determine the 'nominal' detector position.

\section{A3. Reference coordinate system}

In order to determine the longitudinal and transverse components of the resolution function, we define directions parallel and perpendicular to the vector $\mathbf{Q}_{0}$ [equation (40)] (see Fig. 2):

$$
\hat{q}_{\mathrm{rock}}=\hat{q}_{\mathrm{roll}} \times \hat{q}_{\|}=\left[\begin{array}{c}
\cos (\theta) \\
-\sin (\eta) \sin (\theta) \\
\cos (\eta) \sin (\theta)
\end{array}\right]_{\mathrm{lab}},
$$

$$
\begin{gathered}
\hat{q}_{\text {roll }}=\frac{\hat{q}_{\|} \times \hat{k}_{\text {in }}}{k \cos (\theta)}=\left[\begin{array}{c}
0 \\
\cos (\eta) \\
\sin (\eta)
\end{array}\right]_{\mathrm{lab}}, \\
\hat{q}_{\|}=\frac{\mathbf{Q}_{0}}{\left|\mathbf{Q}_{0}\right|}=\left[\begin{array}{c}
-\sin (\theta) \\
-\sin (\eta) \cos (\theta) \\
\cos (\eta) \cos (\theta)
\end{array}\right]_{\mathrm{lab}} .
\end{gathered}
$$

Under nominal working conditions, $\eta=0$, the 'roll' direction is along the laboratory $y$ axis, $\hat{q}_{\text {roll }}=\hat{q}_{\text {lab }}$, whereas $\hat{q}_{\|}$and $\hat{q}_{\text {rock }}$ are within the $(x z)_{\text {lab }}$ plane, and the reference coordinate system is inclined by $\theta_{\mathrm{B}}$ (left-handed rotation about $y$ ) with respect to the laboratory coordinate system.

\section{A4. Reciprocal-space resolution}

We consider the reciprocal-space resolution by adding small perturbations to the horizontal and vertical components of the incident $\left(\Delta \zeta_{\mathrm{h}, \mathrm{v}}\right)$ beam vector, and to the components parallel and perpendicular to the scattering plane $\left(\Delta \xi_{\perp, \|}\right)$ of the scattered beam vector. Furthermore, the beam will have a finite bandwidth. We treat this in the form of a longitudinal perturbation, $\varepsilon=\Delta E / E=\Delta k / k$, to both wavevectors.

Note that $\Delta \zeta_{\mathrm{h}, \mathrm{v}}$ and $\Delta \xi_{\|, \perp}$ are uncorrelated, whereas the longitudinal perturbations are fully correlated for elastic scattering as considered here. In the laboratory coordinate system, the deviations from the nominal incident and diffracted wavevectors are thus

$$
\Delta \mathbf{k}_{\mathrm{in}}=k\left(\begin{array}{c}
\varepsilon \\
\Delta \zeta_{\mathrm{h}} \\
\Delta \zeta_{\mathrm{v}}
\end{array}\right)_{\mathrm{lab}}
$$

$$
\begin{aligned}
\Delta \mathbf{k}_{\text {out }} & =k \mathbf{R}_{x}(\eta) \mathbf{R}_{y}(-2 \theta)\left(\begin{array}{c}
\varepsilon \\
\Delta \xi_{\perp} \\
\Delta \xi_{\|}
\end{array}\right)_{\mathrm{lab}} \\
& =k\left\{\begin{array}{c}
\cos (2 \theta) \varepsilon-\sin (2 \theta) \Delta \xi_{\|} \\
\cos (\eta) \Delta \xi_{\perp}-\sin (\eta)\left[\cos (2 \theta) \Delta \xi_{\|}+\sin (2 \theta) \varepsilon\right] \\
\sin (\eta) \Delta \xi_{\perp}+\cos (\eta)\left[\cos (2 \theta) \Delta \xi_{\|}+\sin (2 \theta) \varepsilon\right]
\end{array}\right\}_{\mathrm{lab}}
\end{aligned}
$$

yielding the deviation from the nominal scattering vector 


$$
\begin{aligned}
& \Delta \mathbf{Q}=\Delta \mathbf{k}_{\text {out }}-\Delta \mathbf{k}_{\text {in }} \\
& =k\left\{\begin{array}{c}
{[\cos (2 \theta)-1] \varepsilon-\sin (2 \theta) \Delta \xi_{\|}} \\
\cos (\eta) \Delta \xi_{\perp}-\sin (\eta)\left[\cos (2 \theta) \Delta \xi_{\|}+\sin (2 \theta) \varepsilon\right]-\Delta \zeta_{\mathrm{h}} \\
\sin (\eta) \Delta \xi_{\perp}+\cos (\eta)\left[\cos (2 \theta) \Delta \xi_{\|}+\sin (2 \theta) \varepsilon\right]-\Delta \zeta_{\mathrm{v}}
\end{array}\right\} .
\end{aligned}
$$

Finally, we express equation (48) in terms of the reference directions [equations (41)-(43)]:

$$
\begin{gathered}
\Delta Q_{\text {rock }}=\Delta \mathbf{Q} \cdot \hat{q}_{\text {rock }} \\
=-\frac{\left|\mathbf{Q}_{0}\right|}{2}\left(\Delta \zeta_{\|}+\Delta \xi_{\|}\right), \\
\Delta Q_{\text {roll }}=\Delta \mathbf{Q} \cdot \hat{q}_{\text {roll }} \\
=-\frac{\left|\mathbf{Q}_{0}\right|}{2 \sin (\theta)}\left(\Delta \zeta_{\perp}-\Delta \xi_{\perp}\right), \\
\Delta Q_{\|}=\Delta \mathbf{Q} \cdot \hat{q}_{\|} \\
=\frac{\left|\mathbf{Q}_{0}\right|}{2}\left[2 \varepsilon+\cot (\theta)\left(-\Delta \zeta_{\|}+\Delta \xi_{\|}\right)\right],
\end{gathered}
$$

where $\quad\left|\mathbf{Q}_{0}\right|=2 k \sin (\theta)$. Furthermore, $\Delta \zeta_{\|}=\cos (\eta) \Delta \zeta_{\mathrm{v}}-$ $\sin (\eta) \Delta \zeta_{\mathrm{h}}$ is the projection of the incident beam's divergence onto the scattering plane, and $\Delta \zeta_{\perp}=\sin (\eta) \Delta \zeta_{\mathrm{v}}+\cos (\eta) \Delta \zeta_{\mathrm{h}}$ is the projection perpendicular to the scattering plane. Note that the result depends on the detector position only and is independent of the sample angles.

At the nominal working point, $\eta=0$, the expressions simplify to $\left(\Delta \zeta_{\perp, \|}=\Delta \zeta_{\mathrm{h}, \mathrm{v}}\right)$

$$
\begin{gathered}
\Delta Q_{\text {rock }}=-\frac{\left|\mathbf{Q}_{0}\right|}{2}\left(\Delta \zeta_{\mathrm{v}}+\Delta \xi_{\|}\right), \\
\Delta Q_{\text {roll }}=-\frac{\left|\mathbf{Q}_{0}\right|}{2 \sin (\theta)}\left(\Delta \zeta_{\mathrm{h}}-\Delta \xi_{\perp}\right), \\
\Delta Q_{\|}=\frac{\left|\mathbf{Q}_{0}\right|}{2}\left[2 \varepsilon+\cot (\theta)\left(\Delta \zeta_{\mathrm{v}}+\Delta \xi_{\|}\right)\right] .
\end{gathered}
$$

When $\Delta \xi$ and $\Delta \zeta$ describe the width of a statistical distribution, e.g. the Gaussian profile of a CRL's acceptance, then the corresponding terms should be added in quadrature to estimate the width in reciprocal space:

$$
\begin{gathered}
\left(\Delta Q_{\text {rock }}\right)^{2}=\frac{\left|\mathbf{Q}_{0}\right|^{2}}{4}\left[\left(\Delta \zeta_{\mathrm{v}}\right)^{2}+\left(\Delta \xi_{\|}\right)^{2}\right], \\
\left(\Delta Q_{\text {roll }}\right)^{2}=\frac{\left|\mathbf{Q}_{0}\right|^{2}}{4 \sin ^{2}(\theta)}\left[\left(\Delta \zeta_{\mathrm{h}}\right)^{2}+\left(\Delta \xi_{\perp}\right)^{2}\right], \\
\left(Q_{\|}\right)^{2}=\frac{\left|\mathbf{Q}_{0}\right|^{2}}{4}\left\{4 \varepsilon^{2}+\cot ^{2}(\theta)\left[\left(\Delta \zeta_{\mathrm{v}}\right)^{2}+\left(\Delta \xi_{\|}\right)^{2}\right]\right\} .
\end{gathered}
$$

These equations can be used for either r.m.s. or FWHM widths as long as one or the other is used consistently.

In particular, when the scattered beam acceptance is given by the Gauss aperture with r.m.s. width $\sigma_{\mathrm{a}}$ [equation (7)], $\Delta \xi_{\mathrm{h}, \mathrm{v}}=\Delta \xi_{\|, \perp}=\sigma_{\mathrm{a}}$ and

$$
\begin{gathered}
\left(\Delta Q_{\text {rock }}\right)^{2}=\frac{\left|\mathbf{Q}_{0}\right|^{2}}{4}\left[\left(\Delta \zeta_{\mathrm{v}}\right)^{2}+\sigma_{\mathrm{a}}^{2}\right], \\
\left(\Delta Q_{\mathrm{roll}}\right)^{2}=\frac{\left|\mathbf{Q}_{0}\right|^{2}}{4 \sin ^{2}(\theta)}\left[\left(\Delta \zeta_{\mathrm{h}}\right)^{2}+\sigma_{\mathrm{a}}^{2}\right],
\end{gathered}
$$

$$
\left(\Delta Q_{\|}\right)^{2}=\frac{\left|\mathbf{Q}_{0}\right|^{2}}{4}\left\{4 \varepsilon^{2}+\cot ^{2}(\theta)\left[\left(\Delta \zeta_{\mathrm{v}}\right)^{2}+\sigma_{\mathrm{a}}^{2}\right]\right\} .
$$

Note that the equations given above describe contributions to the resolution function in the reference coordinate system. For highly asymmetric cases, for example a tightly collimated incident beam and an objective with large numerical aperture, it is more natural to describe the resolution in a coordinate system parallel and perpendicular to the scattered beam axis (see Fig. 8).

By inspecting Fig. 8, we see that the corresponding directions should be perpendicular $\left(\hat{q}_{2 \theta}\right)$ and parallel $\left(\hat{q}_{\text {rock' }}\right)$ to $\hat{k}_{\text {out }}$. The $\hat{q}_{2 \theta}$ direction corresponds to a change of the scattering angle $2 \theta$ and thus to a movement parallel to the surface of the Ewald sphere. The $\hat{q}_{\text {roll }}$ direction is perpendicular to the scattering plane shown in Fig. 8 and therefore also parallel to the Ewald sphere. Consequently, the $\hat{q}_{\text {rock' }}$ direction, being perpendicular to $\hat{q}_{2 \theta}$ and $\hat{q}_{\text {roll }}$, is perpendicular to the surface of the Ewald sphere:

$$
\begin{aligned}
\hat{\boldsymbol{q}}_{2 \theta} & =\frac{1}{k} \frac{\partial \mathbf{Q}}{\partial 2 \theta}=\frac{1}{k} \frac{\partial \mathbf{Q}}{\partial \xi_{\|}}=\left[\begin{array}{c}
-\sin (2 \theta) \\
-\sin (\eta) \cos (2 \theta) \\
\cos (\eta) \cos (2 \theta)
\end{array}\right]_{\mathrm{lab}} \\
& =\cos (\theta) \hat{\boldsymbol{q}}_{\|}-\sin (\theta) \hat{\boldsymbol{q}}_{\mathrm{rock}} .
\end{aligned}
$$

This axis is inclined by $\theta$ with respect to $\hat{q}_{\|}$. Defining the remaining axis to be orthogonal to $\hat{q}_{2 \theta}$,

$$
\begin{aligned}
\hat{q}_{\text {rock' }} & =\sin (\theta) \hat{q}_{\|}+\cos (\theta) \hat{q}_{\text {rock }} \\
& =\left[\begin{array}{c}
\cos (2 \theta) \\
-\sin (\eta) \sin (2 \theta) \\
\cos (\eta) \sin (2 \theta)
\end{array}\right]_{\mathrm{lab}},
\end{aligned}
$$

we find

$$
\Delta Q_{\mathrm{rock}}=\left|\mathbf{Q}_{0}\right|\left[-\cos (\theta) \Delta \zeta_{\|}+\sin (\theta) \varepsilon\right]
$$

Figure 8

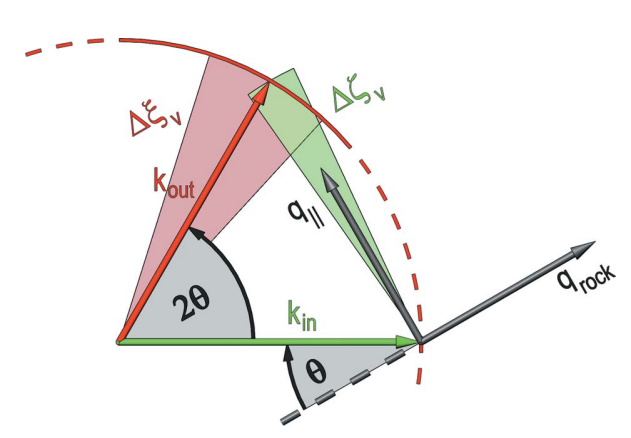

Contribution of the incident beam's divergence, $\Delta \zeta_{\mathrm{v}}$, and objective lens's acceptance, $\Delta \xi_{\mathrm{v}}$, to the in-plane resolution. An offset to the scattering angle $2 \theta$ as given by $\Delta \xi_{\mathrm{v}}$ corresponds to a movement along the surface of the Ewald sphere, indicated by the red dashed line. 


$$
\Delta Q_{2 \theta}=\frac{\left|\mathbf{Q}_{0}\right|}{2 \sin (\theta)}\left[-\cos (2 \theta) \Delta \zeta_{\|}+\sin (2 \theta) \varepsilon+\Delta \xi_{\|}\right] .
$$

At the nominal working point, $\eta=0$, this simplifies to

$$
\begin{gathered}
\Delta Q_{\text {rock }^{\prime}}=\left|\mathbf{Q}_{0}\right|\left[-\cos (\theta) \Delta \zeta_{\mathrm{v}}+\sin (\theta) \varepsilon\right], \\
\Delta Q_{2 \theta}=\frac{\left|\mathbf{Q}_{0}\right|}{2 \sin (\theta)}\left[-\cos (2 \theta) \Delta \zeta_{\mathrm{v}}+\sin (2 \theta) \varepsilon+\Delta \xi_{\|}\right] .
\end{gathered}
$$

Note that $\Delta Q_{\text {rock }^{\prime}}$ does not contain contributions from $\Delta \xi_{\|}$. Thus, for cases with a highly collimated, monochromatic incident beam and an objective with large numerical aperture where $\Delta \zeta_{\mathrm{h}, \mathrm{v}} \simeq \varepsilon \ll \Delta \xi_{\|, \perp}$, the resolution in the $\hat{q}_{\text {rock }^{\prime}}$ direction is much sharper than that in the two other directions, $\Delta Q_{\text {rock }^{\prime}} \ll \Delta Q_{\text {roll }} \simeq \Delta Q_{2 \theta}$.

Again, in the case of statistical distributions, the different contributions are added in quadrature:

$$
\begin{gathered}
\left(\Delta Q_{\text {rock' }}\right)^{2}=\left|\mathbf{Q}_{0}\right|^{2}\left[\cos ^{2}(\theta)\left(\Delta \zeta_{\mathrm{v}}\right)^{2}+\sin ^{2}(\theta) \varepsilon^{2}\right] \\
\left(\Delta Q_{2 \theta}\right)^{2}=\frac{\left|\mathbf{Q}_{0}\right|^{2}}{4 \sin ^{2}(\theta)}\left[\cos ^{2}(2 \theta)\left(\Delta \zeta_{\mathrm{v}}\right)^{2}+\sin ^{2}(2 \theta) \varepsilon^{2}+\left(\Delta \xi_{\|}\right)^{2}\right]
\end{gathered}
$$

\section{A5. Sample}

A reciprocal space vector $\mathbf{G}_{\phi}$ in the $\phi$ coordinate system, rigidly mounted on top of the $\phi$ rotation stage, is then given by

$$
\mathbf{G}_{\phi}=\mathbf{U B}\left(\begin{array}{c}
H \\
K \\
L
\end{array}\right),
$$

where $H, K$ and $L$ are the usual Miller indices, $\mathbf{B}$ describes the reciprocal lattice of the sample, and $\mathbf{U}$ describes the orientation of the sample relative to the $\phi$ coordinate system (Busing \& Levy, 1967; You, 1999).

In the laboratory coordinate system, the same vector is given by

$$
\mathbf{G}_{\mathrm{lab}}=\mathbf{R}_{y}(-\mu) \mathbf{R}_{z}(\omega) \mathbf{R}_{x}(\chi) \mathbf{R}_{y}(-\phi) \mathbf{G}_{\phi},
$$

and the diffraction condition is

$$
\mathbf{Q}=\mathbf{G},
$$

which can be calculated in any chosen coordinate system. We choose to perform the calculation in the $\phi$ coordinate system.

The goniometer settings for a given reciprocal vector $\mathbf{G}_{\phi}$ can be found by back-transforming the scattering vector $\mathbf{Q}$ [equation (40)] into the $\phi$ coordinate system:

$$
\mathbf{Q}_{\phi}=\mathbf{R}_{y}(\phi) \mathbf{R}_{x}(-\chi) \mathbf{R}_{z}(-\omega) \mathbf{R}_{y}(\mu) \mathbf{Q}_{\mathrm{lab}} .
$$

The resulting, lengthy expression is simplified considerably by setting angles to the nominal operating point, $\mu=\theta$ and $\eta=0$ :

$$
\mathbf{Q}_{\phi}=2 k \sin (\theta)\left[\begin{array}{c}
\sin (\phi) \cos (\chi) \\
\sin (\chi) \\
\cos (\phi) \cos (\chi)
\end{array}\right]_{\phi} .
$$

In particular, we see that in this case $\mathbf{Q}_{\phi}$ does not depend on $\omega$, i.e. $\omega$ rotates about the scattering vector, as required for topotomo scans. The goniometer settings for a desired Bragg reflection $\mathbf{G}$ are easily found by setting $\mathbf{Q}_{\phi}=\mathbf{U B G}$ and solving equation (78) for $\chi$ and $\phi$ :

$$
\begin{gathered}
\tan (\phi)=\frac{Q_{\phi, x}}{Q_{\phi, z}}, \\
\tan (\chi)=\frac{Q_{\phi, y}}{\left(Q_{\phi, x}^{2}+Q_{\phi, z}^{2}\right)^{1 / 2}} .
\end{gathered}
$$

The $\operatorname{atan} 2(y, x)$ function can be used instead of $\operatorname{atan}(y / x)$ to avoid division by zero and to determine the correct sectors of $\chi$ and $\phi$. Note, however, that our goniometer implementation restricts both angles to values below $10^{\circ}$.

\section{A6. Practical example: correcting for nonzero $\eta$}

A case that often occurs in practice is that a reflection is found at scattering angle $2 \theta=2 \theta_{0}$ but at nonzero detector azimuth $\eta=\eta_{0} \neq 0$ and for arbitrary positions of $\mu=\mu_{0}$, $\omega=\omega_{0}, \chi=\chi_{0}$ and $\phi=\phi_{0}$. In order to perform azimuthal scans, the $\omega$ axis has to be made parallel to $\mathbf{G}$, i.e. $\eta=0$ and $\mu=\theta$.

The desired settings of the instrument are found in three steps:

(i) First, we determine the scattering vector, $\mathbf{Q}$, from equation (40).

(ii) Next, $\mathbf{Q}_{\phi}$ is found by transforming this vector into the $\phi$ coordinate system using equation (77).

(ii) Finally, the instrument has to be set to $2 \theta=2 \theta_{0}, \eta=0$ and $\mu=2 \theta_{0} / 2$. The remaining angles $\chi$ and $\phi$ are given by equations (79) and (80) using the vector $\mathbf{Q}_{\phi}$ determined above.

Note that this procedure can be carried out without knowing the UB matrix, and even without knowing $k$. It does, however, rely on the correct zero positions of all angles.

\section{A7. Scanning}

In order to measure reciprocal-space-filling non-overlapping maps of intensity, the instrument angles have to be scanned - ideally along mutually perpendicular axes.

Here we distinguish two cases:

Pole figure maps. Pole figures are obtained by scanning along mutually perpendicular sample axes while the detector is kept at the scattering angle $2 \theta$ corresponding to the chosen Bragg reflection. Typical choices for the sample axes are $\chi$ and $\phi$, or $\mu$ and a combined movement of $\chi$ and $\phi-$ the step size in the latter can be adjusted to take into account the asymmetry of the resolution function. As the scans are performed at fixed $|\mathbf{Q}|, \hat{q}_{\text {rock }}$ and $\hat{q}_{\text {roll }}$ can be used as basis.

Three-dimensional reciprocal-space maps. In addition to the sample's orientation, the scattering angle is scanned to monitor the stress-strain state of the sample. As we have seen 
above, the resolution is highly asymmetric. Therefore $\hat{q}_{\mathrm{rock}^{\prime}}$, $\hat{q}_{\text {roll }}$ and $\hat{q}_{2 \theta}$ form a convenient basis for three-dimensional reciprocal-space scans.

We start by projecting the scan directions of the individual instrument axes onto the two reference coordinate systems mentioned above. To leading order, these are given by the partial derivatives of $\mathbf{G}_{\mathrm{lab}}$ with respect to the sample angles, $\mu$, $\omega, \chi$ and $\phi$ :

$$
\begin{gathered}
\frac{1}{\left|\mathbf{Q}_{0}\right|} \frac{\partial \mathbf{G}}{\partial 2 \theta}=\frac{1}{2}\left[\cot (\theta) \hat{q}_{\|}-\hat{q}_{\text {rock }}\right] \\
=\frac{1}{2 \sin (\theta)} \hat{q}_{2 \theta}, \\
\frac{1}{\left|\mathbf{Q}_{0}\right|} \frac{\partial \mathbf{G}}{\partial \eta}=-\cos (\theta) \hat{q}_{\text {roll }}, \\
\frac{1}{\left|\mathbf{Q}_{0}\right|} \frac{\partial \mathbf{Q}}{\partial \mu}=\cos (\eta) \hat{q}_{\text {rock }}+\sin (\eta) \sin (\theta) \hat{q}_{\text {roll }},
\end{gathered}
$$

$$
\begin{aligned}
\frac{1}{\left|\mathbf{Q}_{0}\right|} \frac{\partial \mathbf{Q}}{\partial \omega}= & -\sin (\eta) \cos (\mu) \hat{q}_{\text {rock }} \\
& +[\cos (\eta) \cos (\mu) \sin (\theta)-\sin (\mu) \cos (\theta)] \hat{q}_{\text {roll }},
\end{aligned}
$$

$$
\begin{aligned}
\frac{1}{\left|\mathbf{Q}_{0}\right|} \frac{\partial \mathbf{Q}}{\partial \chi}= & {[-\cos (\omega) \sin (\eta) \sin (\mu)-\cos (\eta) \sin (\omega)] \hat{q}_{\text {rock }} } \\
& +\{\cos (\theta) \cos (\mu) \cos (\omega) \\
& +\sin (\theta)[\cos (\eta) \cos (\omega) \sin (\mu)-\sin (\eta) \sin (\omega)]\} \hat{q}_{\text {roll }}
\end{aligned}
$$

$$
\begin{aligned}
\frac{1}{\left|\mathbf{Q}_{0}\right|} \frac{\partial \mathbf{Q}}{\partial \phi}= & \{\cos (\eta) \cos (\chi) \cos (\omega)+\sin (\eta)[\cos (\mu) \sin (\chi) \\
& -\cos (\chi) \sin (\mu) \sin (\omega)]\} \hat{q}_{\text {rock }} \\
& +\{\sin (\theta)([-\cos (\eta) \cos (\mu)+\cot (\theta) \sin (\mu)] \sin (\chi) \\
& +\cos (\omega) \sin (\eta)+\sin (\omega)[\cos (\mu) \cot (\theta) \\
& +\cos (\eta) \sin (\mu)]\} \cos (\chi)) \hat{q}_{\text {roll }}
\end{aligned}
$$

which simplify at the nominal working point $(\mu=\theta$ and $\eta=0)$ to

$$
\begin{gathered}
\frac{1}{\left|\mathbf{Q}_{0}\right|} \frac{\partial \mathbf{Q}}{\partial 2 \theta}=\frac{1}{2}\left[\cot (\theta) \hat{q}_{\|}-\hat{q}_{\text {rock }}\right] \\
=\frac{1}{2 \sin (\theta)} \hat{\boldsymbol{q}}_{2 \theta}, \\
\frac{1}{\left|\mathbf{Q}_{0}\right|} \frac{\partial \mathbf{Q}}{\partial \eta}=-\cos (\theta) \hat{q}_{\text {roll }}, \\
\frac{1}{\left|\mathbf{Q}_{0}\right|} \frac{\partial \mathbf{Q}}{\partial \mu}=\hat{q}_{\text {rock }}, \\
\frac{1}{\left|\mathbf{Q}_{0}\right|} \frac{\partial \mathbf{Q}}{\partial \omega}=0,
\end{gathered}
$$

$$
\begin{gathered}
\frac{1}{\left|\mathbf{Q}_{0}\right|} \frac{\partial \mathbf{Q}}{\partial \chi}=-\sin (\omega) \hat{q}_{\text {rock }}+\cos (\omega) \hat{q}_{\text {roll }}, \\
\frac{1}{\left|\mathbf{Q}_{0}\right|} \frac{\partial \mathbf{Q}}{\partial \phi}=\cos (\chi)\left[\cos (\omega) \hat{q}_{\text {rock }}+\sin (\omega) \hat{q}_{\text {roll }}\right] .
\end{gathered}
$$

We see in particular that $(\partial \mathbf{G} / \partial \omega)$ vanishes for $\mu=\theta$ and $\eta=0$ as expected, as under nominal working conditions $\omega$ does not change the scattering vector.

Regrouping these contributions by reference direction, the variation of the scattering vector due to small angular changes is then $\left(\Delta \mathbf{Q}=\Delta Q_{\text {rock }} \hat{q}_{\text {rock }}+\Delta Q_{\text {roll }} \hat{q}_{\text {roll }}+\Delta Q_{\|} \hat{q}_{\|}=\right.$ $\left.\Delta Q_{\text {rock }^{\prime}} \hat{q}_{\text {rock }^{\prime}}+\Delta Q_{\text {roll }} \hat{q}_{\text {roll }}+\Delta Q_{2 \theta} \hat{q}_{2 \theta}\right)$,

$$
\begin{aligned}
& \frac{\Delta Q_{\text {rock }}}{\left|\mathbf{Q}_{0}\right|}=-\frac{1}{2}\left(2 \theta-2 \theta_{0}\right)+\left(\mu-\mu_{0}\right) \\
&-\sin \left(\omega_{0}\right)\left(\chi-\chi_{0}\right)+\cos \left(\chi_{0}\right) \cos \left(\omega_{0}\right)\left(\phi-\phi_{0}\right) \\
& \frac{\Delta Q_{\text {roll }}}{\left|\mathbf{Q}_{0}\right|}=-\cos \left(\theta_{0}\right)\left(\eta-\eta_{0}\right)+\cos \left(\omega_{0}\right)\left(\chi-\chi_{0}\right) \\
&+\cos \left(\chi_{0}\right) \sin \left(\omega_{0}\right)\left(\phi-\phi_{0}\right) \\
& \frac{\Delta Q_{\|}}{\left|\mathbf{Q}_{0}\right|}=\frac{1}{2} \cot \left(\theta_{0}\right)\left(2 \theta-2 \theta_{0}\right) \\
& \frac{\Delta Q_{\text {rock }^{\prime}}}{\left|\mathbf{Q}_{0}\right|}=\cos \left(\theta_{0}\right)\left[\left(\mu-\mu_{0}\right)-\sin \left(\omega_{0}\right)\left(\chi-\chi_{0}\right)\right. \\
&\left.+\cos \left(\chi_{0}\right) \cos \left(\omega_{0}\right)\left(\phi-\phi_{0}\right)\right] \\
& \frac{\Delta Q_{2 \theta}}{\left|\mathbf{Q}_{0}\right|}=\frac{1}{2 \sin \left(\theta_{0}\right)}\left(2 \theta-2 \theta_{0}\right)
\end{aligned}
$$

We noted above that under certain experimental conditions the resolution along $\hat{q}_{\text {rock }}$ is much sharper than that along $\hat{q}_{\text {roll }}$. In this case, it is best to perform scans along these directions, i.e. to scan $\mu$ along $\hat{q}_{\text {rock }}$, and a linear combination of $\chi$ and $\phi$ along $\hat{q}_{\text {roll }}$. This can be achieved by a two-motor scan with (using $\mu=\theta$ and $\eta=0$ )

$$
\begin{aligned}
& \Delta \chi=\cos (\omega) \frac{\Delta Q_{\text {roll }}}{\left|\mathbf{Q}_{0}\right|}, \\
& \Delta \phi=\frac{\sin (\omega)}{\cos (\chi)} \frac{\Delta Q_{\text {roll }}}{\left|\mathbf{Q}_{0}\right|} .
\end{aligned}
$$

\section{A8. Relation between angles $\chi$ and $\eta$}

As shown in Fig. 9, the scattering vector $\mathbf{Q}$ will move away from the Ewald sphere (green) when rotating in $\chi$. Let the nearest point on the Ewald sphere be $\mathbf{H}$. With these definitions

$$
\mathbf{H}=\left[\begin{array}{c}
-\sin \left(\theta_{\mathrm{B}}\right) \\
-\cos \left(\theta_{\mathrm{B}}\right) \sin (\eta) \\
\cos \left(\theta_{\mathrm{B}}\right) \cos (\eta)
\end{array}\right],
$$




$$
\begin{aligned}
\mathbf{Q} & =\left[\begin{array}{ccc}
\cos \left(\theta_{\mathrm{B}}\right) & 0 & \sin \left(\theta_{\mathrm{B}}\right) \\
0 & 1 & 0 \\
-\sin \left(\theta_{\mathrm{B}}\right) & 0 & \cos \left(\theta_{\mathrm{B}}\right)
\end{array}\right]\left[\begin{array}{c}
0 \\
\sin (\chi) \\
\cos (\chi)
\end{array}\right] \\
& =\left[\begin{array}{c}
\sin \left(\theta_{\mathrm{B}}\right) \cos (\chi) \\
-\sin (\chi) \\
\cos \left(\theta_{\mathrm{B}}\right) \cos (\chi)
\end{array}\right] .
\end{aligned}
$$

Hence,

$$
\begin{aligned}
\cos (\phi) & =\mathbf{H} \cdot \mathbf{Q} \\
& =\sin ^{2}\left(\theta_{\mathrm{B}}\right) \cos (\chi)+\cos \left(\theta_{\mathrm{B}}\right) \sin ^{2}(\chi)+\cos ^{2}\left(\theta_{\mathrm{B}}\right) \cos ^{2}(\chi) .
\end{aligned}
$$

Expansion to second order gives

$$
\phi=\left[1-\cos \left(\theta_{\mathrm{B}}\right)\right]|\chi| .
$$

\section{A9. Imaging geometry}

Following the discussion of beams through the centre of rotation of the instrument and along the optical axis of the objective lens, we now turn to the mathematical treatment of the imaging geometry.

We consider a new coordinate systems for volume elements within the sample and pixels on the detector. This coordinate system originates at the centre of rotation of the goniometer. The $x$ axis is taken to be along the diffracted beam direction $\mathbf{k}_{\text {out }}$ [equation (39)], and $y$ is perpendicular to the scattering plane, as $\hat{q}_{\text {roll }}$ of equation (42) (see Fig. 10).

$$
\hat{x}_{\mathrm{s}}=\frac{1}{k} \mathbf{k}_{\text {out }}=\left[\begin{array}{c}
\cos (2 \theta) \\
-\sin (\eta) \sin (2 \theta) \\
\cos (\eta) \sin (2 \theta)
\end{array}\right]_{\mathrm{lab}},
$$

$$
\hat{y}_{\mathrm{s}}=\hat{q}_{\mathrm{roll}}=\left[\begin{array}{c}
0 \\
\cos (\eta) \\
\sin (\eta)
\end{array}\right]_{\mathrm{lab}},
$$

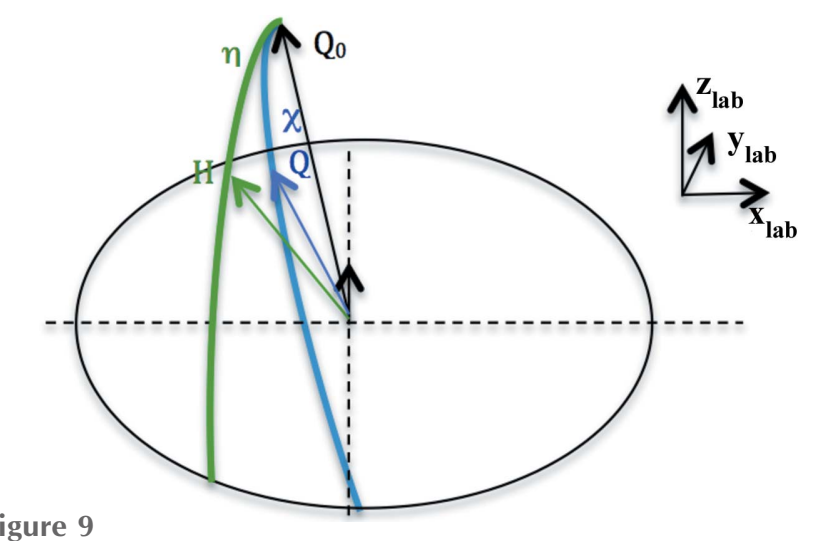

Figure 9

Relation between scattering vector $\mathbf{Q}$ and closest vector on the Ewald sphere $\mathbf{H}$, when scanning in $\chi$. See text.

$$
\hat{z}_{\mathrm{s}}=\hat{x}_{\mathrm{s}} \times \hat{y}_{\mathrm{s}}=\left[\begin{array}{c}
-\sin (2 \theta) \\
-\sin (\eta) \cos (2 \theta) \\
\cos (\eta) \cos (2 \theta)
\end{array}\right]_{\mathrm{lab}} .
$$

Voxels within the sample plane at $\mathbf{r}_{\mathrm{s}}=\left(0, y_{\mathrm{s}}, z_{\mathrm{s}}\right)$ are projected onto detector pixels at $\mathbf{r}_{\mathrm{d}}=\left(L,-\mathcal{M} y_{\mathrm{s}},-\mathcal{M} z_{\mathrm{s}}\right)$, where $L$ is the distance from the origin to the detector plane and $\mathcal{M}=\mathcal{M}_{\mathrm{CRL}} \mathcal{M}_{\mathrm{d}}$ is the overall magnification of the system. Alternatively, the positions $y_{\mathrm{d}}$ and $z_{\mathrm{d}}$ can be measured on the scintillator screen of the detector, without taking into account the visual magnification $\mathcal{M}_{\mathrm{d}}$. In this case $\mathcal{M}=\mathcal{M}_{\mathrm{CRL}}$.

For small $x_{\mathrm{s}} \ll d_{1}$ and sufficient depth of field (see main text), this can also be assumed for voxels outside of the sample plane $x_{\mathrm{s}} \neq 0$. Note, however, that this may not be the case for objective lenses with very short working distance $d_{1}$ and large numerical aperture.

Following Simons et al. (2017), we note that rays connecting these two points pass through the objective lens at an angular offset,

$$
\begin{aligned}
& \Delta \xi_{\perp}=-\gamma y_{\mathrm{s}}=-\frac{\gamma}{\mathcal{M}} y_{\mathrm{d}}, \\
& \Delta \xi_{\|}=-\gamma z_{\mathrm{s}}=-\frac{\gamma}{\mathcal{M}} z_{\mathrm{d}},
\end{aligned}
$$

leading to an offset in the scattering vector as a function of detector pixel,

$$
\begin{aligned}
\mathbf{Q}_{\text {shift }} & =\frac{k \gamma}{\mathcal{M}}\left[\begin{array}{c}
\sin (2 \theta) z_{\mathrm{d}} \\
-\cos (\eta) y_{\mathrm{d}}+\cos (2 \theta) \sin (\eta) z_{\mathrm{d}} \\
-\sin (\eta) y_{\mathrm{d}}-\cos (2 \theta) \cos (\eta) z_{\mathrm{d}}
\end{array}\right]_{\mathrm{lab}} \\
& =\frac{\gamma\left|\mathbf{Q}_{0}\right|}{2 \mathcal{M}}\left[\cot (\theta) z_{\mathrm{d}}\left(\hat{q}_{\text {rock }}-\hat{\boldsymbol{q}}_{\|}\right)-\frac{1}{\sin (\theta)} y_{\mathrm{d}} \hat{\boldsymbol{q}}_{\mathrm{roll}}\right] .
\end{aligned}
$$

In other words

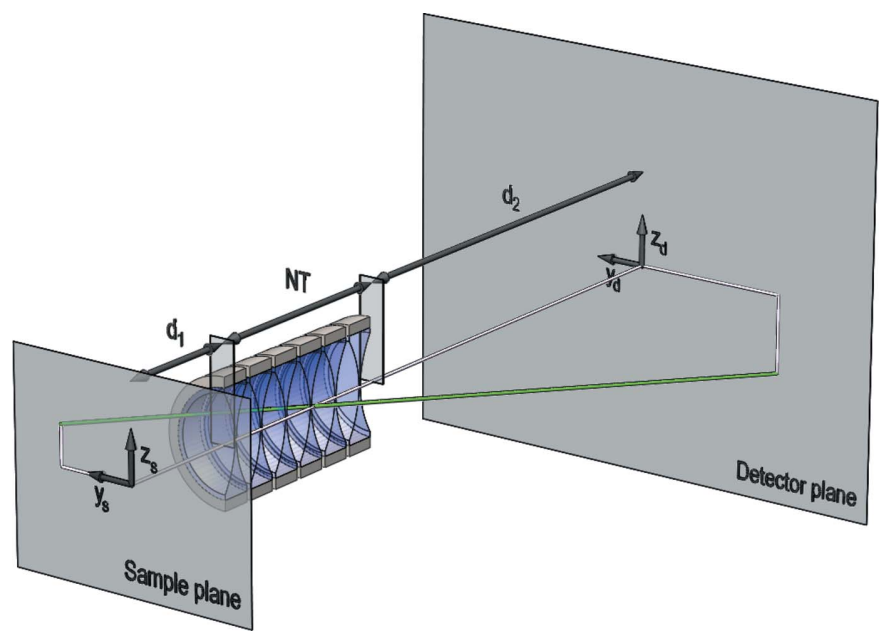

Figure 10

Sample and detector coordinates. $x$ is along the diffracted beam axis, and $y$ is perpendicular to the scattering plane. A voxel at position $\mathbf{r}_{\mathrm{s}}=\left(x_{\mathrm{s}} \simeq 0, y_{\mathrm{s}}, z_{\mathrm{s}}\right)$ is projected onto the detector pixel at $\mathbf{r}_{\mathrm{d}}=L \hat{x}-\mathcal{M} \mathbf{r}_{\mathrm{s}}=\left(L, y_{\mathrm{d}}, z_{\mathrm{d}}\right)$, where $L$ is the distance from the centre of rotation to the detector plane. 


$$
\begin{aligned}
& \frac{\Delta Q_{\text {shift,rock }}}{\left|\mathbf{Q}_{0}\right|}=\frac{\gamma}{2 \mathcal{M}} \cot (\theta) z_{\mathrm{d}}, \\
& \frac{\Delta Q_{\text {shift,roll }}}{\left|\mathbf{Q}_{0}\right|}=-\frac{\gamma}{2 \mathcal{M}} \frac{1}{\sin (\theta)} y_{\mathrm{d}}, \\
& \frac{\Delta Q_{\text {shift }, \|}}{\left|\mathbf{Q}_{0}\right|}=-\frac{\gamma}{2 \mathcal{M}} \cot (\theta) z_{\mathrm{d}}
\end{aligned}
$$

\section{Acknowledgements}

The authors are grateful to Stefan Poulsen, Frederik Stöhr, Anders Filsoe Pedersen and Jose Xavier Trujillo for stimulating discussions. We thank the ESRF for beamtime and the instrument centre DANSCATT for support of travel. We thank Ragnvald Mathiesen as well as the anonymous referees for excellent comments on the paper. HFP and SRA acknowledge support by the ERC grant 'Diffraction Based Transmission X-ray Microscopy', and HS support by a DFFFTP individual postdoc grant.

\section{References}

Ahl, S. R., Simons, H., Jakobsen, A. C., Zhang, Y. B., Stöhr, F., Jensen, D. J. \& Poulsen, H. F. (2015). Mater. Sci. Eng. 89, 012016.

Als-Nielsen, J. \& McMorrow, D. (2011). Elements of Modern X-ray Physics. Chichester: Wiley.

Bergbäck Knudsen, E. et al. (2013). J. Appl. Cryst. 46, 679-696.

Busing, W. R. \& Levy, H. A. (1967). Acta Cryst. 22, 457-464.

Cereser, A. et al. (2017). Nat Sci. Rep. 7, 9561.

Falch, K. V., Casari, D., Di Michiel, M., Detlefs, C., Snigireva, A., Snigireva, I., Honkimäki, V. \& Mathiesen, R. H. (2017). J. Mater. Sci. 52, 3497-3507.

Falch, K. V., Lyubomirskij, M., Casari, D., Detlefs, C., Snigirev, A., Snigireva, I., Di Michiel, M., Honkimaki, V. \& Mathiesen, R. H. (2017). Ultramicroscopy. Submitted.

Hefferan, C. M., Lind, J., Li, S. F., Lienert, U., Rollett, A. D. \& Suter, R. M. (2012). Acta Mater. 60, 4311-4318.

Hutchings, M. T., Withers, P. J., Holden, T. M. \& Lorentzen, T. (2005). Introduction to the Characterization of Residual Stress by Neutron Diffraction. Boca Raton: CRC Press.

Jakobsen, A. et al. (2017). Dark-Field X-ray Microscopy of Dislocations in Single-Crystal Diamond. In preparation.

Kak, A. C. \& Slaney, M. (1988). Principles of Computerized Tomographic Imaging. New York: IEEE Press.

King, A., Johnson, G., Engelberg, D., Ludwig, W. \& Marrow, J. (2008). Science, 321, 382-385.

Koch, A., Raven, C., Spanne, P. \& Snigirev, A. (1998). J. Opt. Soc. Am. A, 15, 1940-1951.
Kohn, V. G. (2002). JETP Lett. 76, 600-603.

Krywka, C., Last, A., Marschall, F., Márkus, O., Georgi, S., Müller, M. \& Mohr, J. (2016). AIP Conf. Proc. 1764, 020001.

Lengeler, B., Schroer, C. G., Richwin, M., Tümmler, J., Drakopoulos, M., Snigirev, A. \& Snigireva, I. (1999). Appl. Phys. Lett. 74, 39243926.

Ludwig, W., Cloetens, P., Härtwig, J., Baruchel, J., Hamelin, B. \& Bastie, P. (2001). J. Appl. Cryst. 34, 602-607.

Ludwig, W., Reischig, P., King, A., Herbig, M., Lauridsen, E. M., Johnson, G., Marrow, T. J. \& Buffière, J. Y. (2009). Rev. Sci. Instrum. 80, 033905.

Medrano, C., Rejmánková, P., Ohler, M. \& Matsouli, I. (1997). Nouv. Cim. D, 19, 195-203.

Midgley, P. \& Eggemann, A. S. (2015). Ultramicroscopy, 21, 126-136.

Morgan, A. J., Prasciolu, M., Andrejczuk, A., Krzywinski, J., Meents, A., Pennicard, D., Graafsma, H., Barty, A., Bean, R. J., Barthelmess, M., Oberthuer, D., Yefanov, O., Aquila, A., Chapman, H. N. \& Bajt, S. (2015). Sci. Rep. 5, 9892.

Ohler, M., Sanchez del Rio, M., Tuffanelli, A., Gambaccini, M., Taibi, A., Fantini, A. \& Pareschi, G. (2000). J. Appl. Cryst. 33, 1023-1030.

Peetermans, S., King, A., Ludwig, W., Reischig, P. \& Lehmann, E. H. (2014). Analyst, 139, 5765-5771.

Poulsen, H. F. (2003). Philos. Mag. 83, 2761-2778.

Poulsen, H. F. (2012). J. Appl. Cryst. 45, 1084-1097.

Poulsen, H. F., Nielsen, S. F., Lauridsen, E. M., Schmidt, S., Suter, R. M., Lienert, U., Margulies, L., Lorentzen, T. \& Juul Jensen, D. (2001). J. Appl. Cryst. 34, 751-756.

Poulsen, S. O., Poulsen, H. F. \& Bentley, P. M. (2014). Nucl. Instrum. Methods Phys. Res. Sect. A, 767, 415-420.

Rayleigh, Lord (1891). Philos. Mag. Ser. 5, 31, 87-99.

Richter, T., Seiler, J., Schnurrer, W. \& Kaup, A. (2016). IEEE Trans. Circuits Systems Video Technol. 26, 814-828.

Schmidt, S. (2014). J. Appl. Cryst. 47, 276-284.

Schroer, C. G. \& Lengeler, B. (2005). Phys. Rev. Lett. 94, 054802.

Simons, H., Ahl, S. R., Poulsen, H. F. \& Detlefs, C. (2017). J. Synchrotron Rad. 24, 392-401.

Simons, H., Jakobsen, A. C., Ahl, S. R., Detlefs, C. \& Poulsen, H. F. (2016). MRS Bull. 41, 454-459.

Simons, H., King, A., Ludwig, W., Detlefs, C., Pantleon, W., Schmidt, S., Snigireva, I., Snigirev, A. \& Poulsen, H. F. (2015). Nat. Commun. 6, 6098 .

Simons, H., Stöhr, F., Michael-Lindhard, J., Jensen, F., Hansen, O., Detlefs, C. \& Poulsen, H. F. (2016). Opt. Commun. 359, 460-464.

Snigirev, A., Kohn, V. G., Snigireva, I. I. \& Lengeler, B. (1996). Nature, 384, 49-51.

Sørensen, H., Schmidt, S., Wright, J. P., Vaughan, G. B. M., Techert, S., Garman, E., Oddershede, J., Davaasambuu, J., Paithankar, K. S., Gundlach, C. \& Poulsen, H. F. (2012). Z. Kristallogr. 227, 63-78.

Svelto, O. (2010). Principles of Lasers, 5th ed. New York: Springer.

Vila-Comamala, J., Pan, Y., Lombardo, J., Harris, W. M., Chiu, W. K., David, C. \& Wang, Y. (2012). J. Synchrotron Rad. 19, 705-709.

Vlieg, E. (1997). J. Appl. Cryst. 30, 532-543.

Williams, D. B. \& Carter, C. B. (2009). Transmission Electron Microscopy. Berlin: Springer.

You, H. (1999). J. Appl. Cryst. 32, 614-623. 